Homology, Homotopy and Applications, vol.17(2), 2015, pp.291-316

\title{
THE EXISTENCE OF HOMOTOPY RESOLUTIONS OF $N$-COMPLEXES
}

\author{
XIAOYAN YANG AND JUNPENG WANG
}

(communicated by Donald M. Davis)

\begin{abstract}
In this paper complexes with $N$-nilpotent differentials are considered. We proceed by generalizing a defining property of injective and projective resolutions to define $d g$-injective and $d g$-projective $N$-complexes, and construct $d g$-injective and $d g$ projective resolutions for arbitrary $N$-complexes. As applications of these results, we prove that the category $\mathcal{D}_{N}(R)$ is compactly generated, the category $\mathcal{K}_{N}(\mathscr{I})$ of injectives is compactly generated whenever $R$ is left noetherian, and the category $\mathcal{K}_{N}(\mathscr{P})$ of projectives is compactly generated whenever $R$ is a right coherent ring for which every flat left $R$-module has finite projective dimension. We also establish a recollement of the category $\mathcal{K}_{N}(R)$ relative to $\mathcal{K}_{N}^{e x}(R)$ and $\mathcal{D}_{N}(R)$.
\end{abstract}

\section{Introduction}

Homological algebra mostly studies complexes having a differential $d$ satisfying $d^{2}=0$. It is natural to ask why $d^{2}=0$ and not, say, $d^{3}=0$. The idea to investigate complexes with a differential $d$ such that $d^{N}=0$ where $N \geqslant 3$ was introduced by Kapranov, [11] and there he hinted at their possible connections to quantum theories. Since then many papers have appeared on the subject, many of them studying their interesting homology (recently called "amplitude homology"), pointing to their relevance in theoretical physics, and indicating some possible applications of $N$-complexes for certain nonassociative algebras. In 2013 Gillespie [5] made a brief study of contractible $N$-complexes. In 2015 Yang and Ding [16] provided an effective construction of left and right triangles, and proved that the homotopy category and the derived category of $N$-complexes are pretriangulated categories.

In classical homological algebra the most fundamental concept is that of a resolution of an object. In the theory of derived categories an analogous role is played by resolutions of complexes. Resolutions of bounded complexes can be handled in much the same way as resolutions of objects, but the situation for unbounded complexes is different. Here, we proceed by generalizing a defining property of injective and projective resolutions to define $d g$-injective and $d g$-projective $N$-complexes, and

Received December 2, 2014, revised March 9, 2015, June 24, 2015; published on November 18, 2015. 2010 Mathematics Subject Classification: 18G35, 18E30, 18G10.

Key words and phrases: $N$-complex, homotopy category, homotopy resolution, recollement.

Article available at http://dx.doi.org/10.4310/HHA.2015.v17.n2.a14

Copyright (C) 2015, International Press. Permission to copy for private use granted. 
construct $d g$-injective resolution of any bounded-above $N$-complex and $d g$-projective resolution of any bounded-below $N$-complex. Furthermore, we prove that $d g$-injective and $d g$-projective resolutions for arbitrary $N$-complexes by these special $N$-complexes exist.

There have been some beautiful results, starting in 2005 with Krause [12] and Jøgensen [10], and continuing with Iyengar and Krause [9] and Neeman [15] that focus on the properties of the homotopy category $\mathcal{K}(\mathscr{X})$ in the usual sense $(N=2)$. The results of Krause and Jøgensen proved that for sufficiently nice $\operatorname{rings}, \mathcal{K}(\operatorname{Inj} R)$ and $\mathcal{K}(\operatorname{Prj} R)$ are compactly generated and are infinite completions of $\mathcal{D}^{b}(R)^{\mathrm{op}}$ and $\mathcal{D}^{b}(R)$, respectively. In this paper, we show that the category $\mathcal{D}_{N}(R)$ is compactly generated, the category $\mathcal{K}_{N}(\mathscr{I})$ of injectives is compactly generated whenever $R$ is left noetherian, and the category $\mathcal{K}_{N}(\mathscr{P})$ of projectives is compactly generated whenever $R$ is a right coherent ring for which every flat left $R$-module has finite projective dimension. We also establish a recollement of the pretriangulated category $\mathcal{K}_{N}(R)$ relative to $\mathcal{K}_{N}^{e x}(R)$ and $\mathcal{D}_{N}(R)$.

\section{Preliminaries and basic facts}

Unless otherwise stated, we assume throughout this paper that $\mathscr{A}$ is an abelian category.

This section is devoted to recalling some notions and basic consequences for use throughout this paper. For terminology we shall follow $[\mathbf{1}],[\mathbf{2}]$ and $[\mathbf{3}]$ when working with left, right, and pretriangulated categories, and we shall follow [5] and [16] when working with $N$-complexes.

\section{Left and right triangulated categories.}

Let $\mathscr{T}$ be an additive category and $\Omega: \mathscr{T} \rightarrow \mathscr{T}$ an additive endofunctor. Let $\mathcal{L T}(\mathscr{T}, \Omega)$ denote the category with objects the diagrams in $\mathscr{T}$ of the form $\Omega Z \stackrel{\mu}{\rightarrow}$ $X \stackrel{\nu}{\rightarrow} Y \stackrel{\omega}{\rightarrow} Z$ and with set of morphisms from $\Omega Z_{1} \stackrel{\mu_{1}}{\rightarrow} X_{1} \stackrel{\nu_{1}}{\rightarrow} Y_{1} \stackrel{\omega_{1}}{\rightarrow} Z_{1}$ to $\Omega Z_{2} \stackrel{\mu_{2}}{\rightarrow} X_{2} \stackrel{\nu_{2}}{\rightarrow}$ $Y_{2} \stackrel{\omega_{2}}{\rightarrow} Z_{2}$ the triples $(f, g, h)$ of morphisms in $\mathscr{T}$ from $\left(X_{1}, Y_{1}, Z_{1}\right)$ to $\left(X_{2}, Y_{2}, Z_{2}\right)$, which make the following diagram commutative:

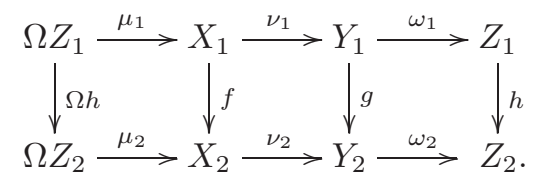

Such a morphism is said to be an isomorphism if $f, g, h$ are isomorphisms in $\mathscr{T}$.

A left triangulation of the pair $(\mathscr{T}, \Omega)$ is a full subcategory $\Delta$ of $\mathcal{L} \mathcal{T}(\mathscr{T}, \Omega)$ that satisfies the following axioms.

(LT1) $\Delta$ is closed under isomorphisms. For every object $X$ in $\mathscr{T}$, the diagram $0 \rightarrow X \stackrel{i d_{x}}{\rightarrow} X \rightarrow 0$ belongs to $\Delta$. For any morphism $\omega: Y \rightarrow Z$, there exists a diagram in $\Delta$ of the form $\Omega Z \stackrel{\mu}{\rightarrow} X \stackrel{\nu}{\rightarrow} Y \stackrel{\omega}{\rightarrow} Z$.

(LT2) For any diagram $\Omega Z \stackrel{\mu}{\rightarrow} X \stackrel{\nu}{\rightarrow} Y \stackrel{\omega}{\rightarrow} Z$ in $\Delta$, the diagram $\Omega Y \stackrel{-\Omega \omega}{\rightarrow} \Omega Z \stackrel{\mu}{\rightarrow} X \stackrel{\nu}{\rightarrow}$ $Y$ is also in $\Delta$. 
(LT3) Given two diagrams $\Omega Z \stackrel{\mu}{\rightarrow} X \stackrel{\nu}{\rightarrow} Y \stackrel{\omega}{\rightarrow} Z$ and $\Omega Z^{\prime} \stackrel{\mu^{\prime}}{\rightarrow} X^{\prime} \stackrel{\nu^{\prime}}{\rightarrow} Y^{\prime} \stackrel{\omega^{\prime}}{\rightarrow} Z^{\prime}$ in $\Delta$, then each commutative diagram

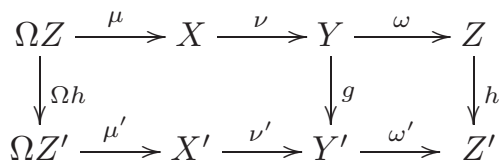

can be completed to a morphism of diagrams in $\Delta$.

(LT4) For any two diagrams $\Omega Z \stackrel{\mu}{\rightarrow} X \stackrel{\nu}{\rightarrow} Y \stackrel{\omega}{\rightarrow} Z$ and $\Omega X^{\prime} \stackrel{\alpha^{\prime}}{\rightarrow} Z^{\prime} \stackrel{\beta^{\prime}}{\rightarrow} Z \stackrel{\gamma^{\prime}}{\rightarrow} X^{\prime}$ in $\Delta$, there exists a commutative diagram

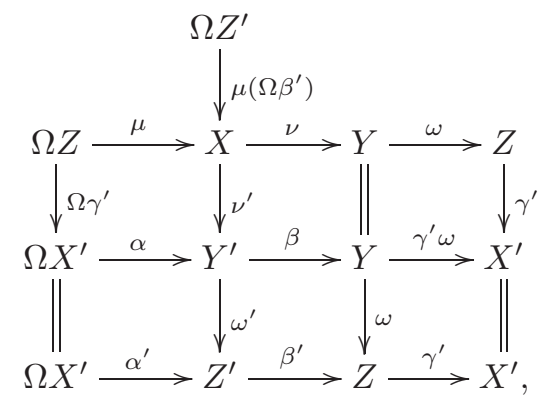

where the middle row and the second column are diagrams in $\Delta$.

Then the triple $(\mathscr{T}, \Omega, \Delta)$ is called a left triangulated category, the functor, $\Omega$ is the loop functor and the diagrams in $\Delta$ are the left triangles.

Dually, let $\mathscr{T}$ be an additive category and $\Sigma: \mathscr{T} \rightarrow \mathscr{T}$ an additive endofunctor. A right triangulation of the pair $(\mathscr{T}, \Sigma)$ is a full subcategory $\nabla$ of $\mathcal{R T}(\mathscr{T}, \Sigma)$ that satisfies the right analogs of axioms (LT1)-(LT4). Then the triple $(\mathscr{T}, \Sigma, \nabla)$ is called a right triangulated category, the functor $\Sigma$ is the suspension functor, and the diagrams in $\nabla$ are the right triangles.

A nice introduction to the basic idea of a left or right triangulated category can be found in [1] and [2].

\section{Pretriangulated categories.}

Let $\mathscr{T}$ be an additive category. A pre-triangulation of $\mathscr{T}$ consists of the following data:

(PT1) An adjoint pair $(\Sigma, \Omega)$ of additive endofunctors $\Sigma, \Omega: \mathscr{T} \rightarrow \mathscr{T}$. Let $\varepsilon$ :

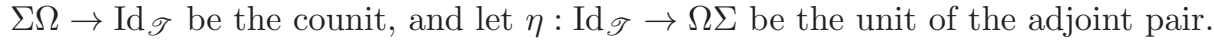

(PT2) A collection of diagrams $\Delta$ in $\mathscr{T}$ of the form $\Omega Z \stackrel{\mu}{\rightarrow} X \stackrel{\nu}{\rightarrow} Y \stackrel{\omega}{\rightarrow} Z$, such that the triple $(\mathscr{T}, \Omega, \Delta)$ is a left triangulated category.

(PT3) A collection of diagrams $\nabla$ in $\mathscr{T}$ of the form $X^{\prime} \stackrel{\mu^{\prime}}{\rightarrow} Y^{\prime} \stackrel{\nu^{\prime}}{\rightarrow} Z^{\prime} \stackrel{\omega^{\prime}}{\rightarrow} \Sigma X^{\prime}$, such that the triple $(\mathscr{T}, \Sigma, \nabla)$ is a right triangulated category.

(PT4) For any diagram in $\mathscr{T}$ with commutative left square:

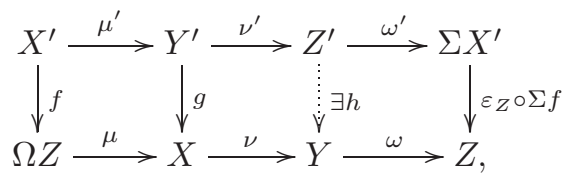


where the upper row is in $\nabla$ and the lower row is in $\Delta$, there exists a morphism $h: Z^{\prime} \rightarrow Y$ making the diagram commutative.

(PT5) For any diagram in $\mathscr{T}$ with commutative right square:

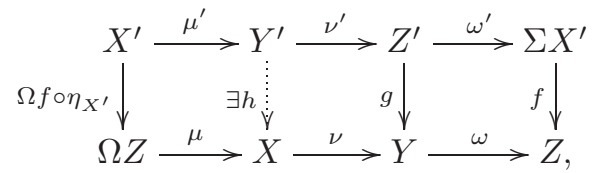

where the upper row is in $\nabla$ and the lower row is in $\Delta$, there exists a morphism $h: Y^{\prime} \rightarrow X$ making the diagram commutative.

A pretriangulated category is an additive category together with a pre-triangulation and is denoted by $\mathscr{T}=(\mathscr{T}, \Sigma, \Omega, \nabla, \Delta, \varepsilon, \eta)$. A nice introduction to the basic idea of a pretriangulated category can be found in [3].

Let $\mathscr{T}$ be a left (resp. right) triangulated category. A full additive subcategory $\mathscr{S}$ in $\mathscr{T}$ is called a left (resp. right) triangulated subcategory if it is replete, if $\Omega \mathscr{S} \subseteq \mathscr{S}$ (resp. $\Sigma \mathscr{S} \subseteq \mathscr{S}$ ), if for any left triangle $\Omega Z \rightarrow X \rightarrow Y \rightarrow Z$ (resp. right triangle $X^{\prime} \rightarrow Y^{\prime} \rightarrow Z^{\prime} \rightarrow \Sigma X^{\prime}$ ) such that $Y, Z$ are in $\mathscr{S}$, the object $X$ is also in $\mathscr{S}$ (resp. such that $X^{\prime}, Y^{\prime}$ are in $\mathscr{S}$, the object $Z^{\prime}$ is also in $\mathscr{S}$ ). Let $\mathscr{T}$ be a pretriangulated category. A full additive subcategory $\mathscr{S}$ in $\mathscr{T}$ is called a pretriangulated subcategory if $\mathscr{S}$ is a left and right triangulated subcategory. A thick subcategory $\mathscr{S}$ of a pretriangulated category $\mathscr{T}$ is a full pretriangulated subcategory of $\mathscr{T}$ such that $\mathscr{S}$ is closed under isomorphism classes and taking direct summands.

\section{$N$-complexes.}

By an $N$-complex $X(N \geqslant 2)$ we mean a sequence of objects in $\mathscr{A}$

$$
\cdots \stackrel{d}{\longrightarrow} X_{n+1} \stackrel{d}{\longrightarrow} X_{n} \stackrel{d}{\longrightarrow} X_{n-1} \stackrel{d}{\longrightarrow} \cdots
$$

satisfying $d^{N}=0$. That is, composing any $N$-consecutive morphisms gives 0 . So a 2 complex is a chain complex in the usual sense. A chain map or simply map $f: X \rightarrow Y$ of $N$-complexes is a collection of morphisms $f_{n}: X_{n} \rightarrow Y_{n}$ making all the rectangles commute. We get a category of $N$-complexes, denoted by $\mathcal{C}_{N}(\mathscr{A})$, whose objects are $N$-complexes and whose morphisms are chain maps. This is an abelian category.

For an $N$-complex $X$, there are $N-1$ choices for homology. Indeed, for $t=$ $1, \cdots, N$, we define $\mathrm{Z}_{n}^{t}(X)=\operatorname{Ker}\left(d_{n-(t-1)} \cdots d_{n-1} d_{n}\right)$ and $\mathrm{B}_{n}^{t}(X)=\operatorname{Im}\left(d_{n+1} d_{n+2} \cdots\right.$ $\left.d_{n+t}\right)$. In particular, we have $\mathrm{Z}_{n}^{1}(X)=\operatorname{Ker} d_{n}, \mathrm{Z}_{n}^{N}(X)=X_{n}$, and $\mathrm{B}_{n}^{1}(X)=\operatorname{Im} d_{n+1}$, $\mathrm{B}_{n}^{N}(X)=0$. We also set $\mathrm{C}_{n}^{t}(X)=X_{n} / \mathrm{B}_{n}^{t}(X)$, and define $\mathrm{H}_{n}^{t}(X)=\mathrm{Z}_{n}^{t}(X) / \mathrm{B}_{n}^{N-t}(X)$ the amplitude homology objects of $X$ for all $t$. We say $X$ is $N$-exact, or just exact, if $\mathrm{H}_{n}^{t}(X)=0$ for all $n$ and $t$. The class of exact $N$-complexes is denoted by $\mathscr{E}_{N}$.

Example 2.1. (1) Let $R$ be a ring, $A_{\infty}^{\infty} \equiv \cdots \rightarrow v_{-1} \rightarrow v_{0} \rightarrow v_{1} \rightarrow \cdots$ the infinite quiver, and $I_{N}$ the ideal of $R A_{\infty}^{\infty}$ generated by all paths of length at least $N(N \geqslant 2)$. The category of $R A_{\infty}^{\infty} / I_{N}$-modules is equivalent to the category of $N$-complexes of modules (see $[4]$ ).

(2) Let $R$ be a ring and $N \geqslant 2$ an fixed integer. The category of graded modules over the graded ring $R[x] /\left(x^{N}\right)$ is isomorphic to the category of $N$-complexes (see $[6])$. 
(3) Let $(X, d)$ be an $N$-complex in $\mathscr{A}$. Define

$$
\begin{gathered}
i_{*}: \mathrm{H}_{n}^{t}(X) \rightarrow \mathrm{H}_{n}^{t+1}(X) \text { via } x+\operatorname{Im} d^{N-t} \mapsto x+\operatorname{Im} d^{N-t-1}, \\
d_{*}: \mathrm{H}_{n}^{t}(X) \rightarrow \mathrm{H}_{n-1}^{t-1}(X) \text { via } x+\operatorname{Im} d^{N-t} \mapsto d(x)+\operatorname{Im} d^{N-t+1} .
\end{gathered}
$$

Moreover, define $\mathbf{H}_{m}(X)=\bigoplus_{2 n-t=m} \mathrm{H}_{n}^{t}(X)$ and define morphisms $D: \mathbf{H}_{m}(X) \rightarrow$ $\mathbf{H}_{m-1}(X)$ by $D=i_{*}+d_{*}$ (with the convention that $i_{*}$ and $d_{*}$ are set to be zero when not defined). Then $(\mathbf{H}(X), D)$ is an $(N-1)$-complex (see [11]).

In general, the differential induces the following poset of non-trivial subobjects where the arrows denote inclusion:

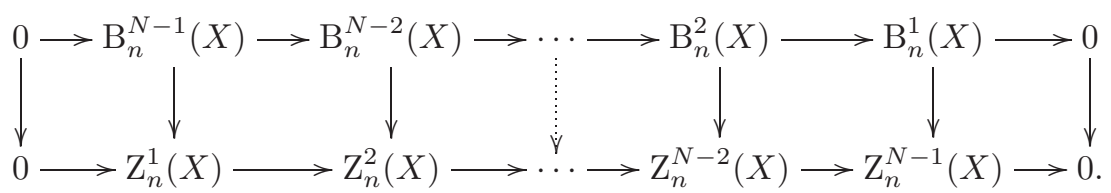

The differential $d$ adds a dimension to diagram (2.1) by inducing the vertical morphisms in

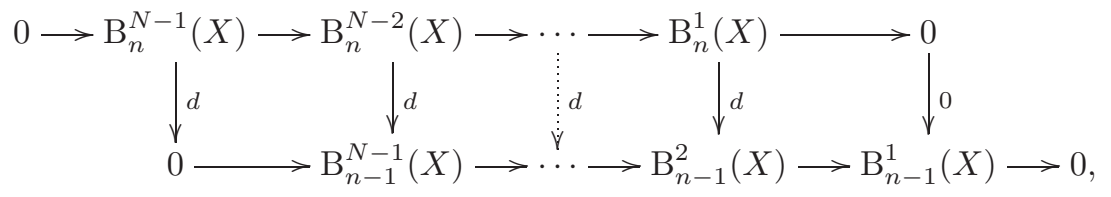

where the rows are coming from the top row of (2.1). The same thing can be done for the bottom row of (2.1):

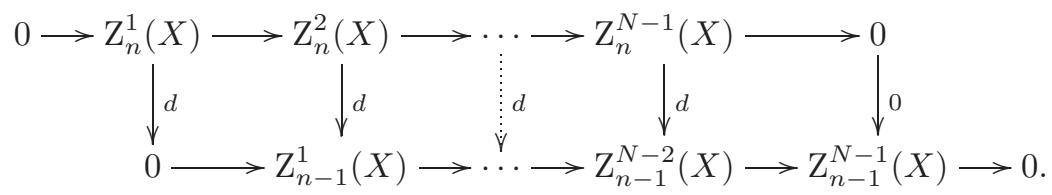

Diagrams (2.2) and (2.3) both commute.

Given an object $A$ of $\mathscr{A}$, we define $N$-complexes $\mathrm{D}_{n}^{t}(A)$ for $t=1, \cdots, N$ as follows. $\mathrm{D}_{n}^{t}(A)$ consists of $A$ in degrees $n, n-1, \cdots, n-(t-1)$, all joined by identity morphisms, and 0 in every other degree. We will call it the disk on $A$ of degree $n$. Let $\left\{M_{n} \mid n \in \mathbb{Z}\right\}$ be objects in $\mathscr{A}$. Then $\left(\coprod_{n \in \mathbb{Z}} \mathrm{D}_{n}^{N}\left(M_{n}\right)\right)_{k}=\left(\prod_{n \in \mathbb{Z}} \mathrm{D}_{n}^{N}\left(M_{n}\right)\right)_{k}=$ $M_{k+N-1} \oplus \cdots \oplus M_{k}$ for all $k$. Therefore,

$$
\coprod_{n \in \mathbb{Z}} \mathrm{D}_{n}^{N}\left(M_{n}\right)=\prod_{n \in \mathbb{Z}} \mathrm{D}_{n}^{N}\left(M_{n}\right) .
$$

Two chain maps $f, g: X \rightarrow Y$ are called chain homotopic, or simply homotopic, if there exists a collection of morphisms $\left\{s_{n}: X_{n} \rightarrow Y_{n+N-1}\right\}$ such that

$$
\begin{gathered}
g_{n}-f_{n}=d^{N-1} s_{n}+d^{N-2} s_{n-1} d+\cdots+s_{n-(N-1)} d^{N-1}= \\
\sum_{i=0}^{N-1} d^{(N-1)-i} s_{n-i} d^{i}, \forall n .
\end{gathered}
$$

If $f$ and $g$ are homotopic, then we write $f \sim g$. We call a chain map $f$ null homotopic if $f \sim 0$. There exists an additive category $\mathcal{K}_{N}(\mathscr{A})$, called the homotopy category of 
$N$-complexes, whose objects are the same as those of $\mathcal{C}_{N}(\mathscr{A})$ and whose Hom sets are the $\sim$ equivalence classes of Hom sets in $\mathcal{C}_{N}(\mathscr{A})$. An isomorphism in $\mathcal{K}_{N}(\mathscr{A})$ is called a homotopy equivalence.

\section{Left and right triangles in $\mathcal{K}_{N}(\mathscr{A})$.}

Let $X$ be an $N$-complex in $\mathcal{C}_{N}(\mathscr{A})$. Define $(\Omega X)_{n}=X_{n+N-1} \oplus \cdots \oplus X_{n+2} \oplus X_{n+1}$ with differential given by

$$
d=\left(\begin{array}{cccccc}
0 & 1 & 0 & \cdots & 0 & 0 \\
\vdots & \vdots & \vdots & \vdots & \vdots & \vdots \\
0 & 0 & 0 & \cdots & 0 & 1 \\
-d^{N-1} & -d^{N-2} & -d^{N-3} & \cdots & -d^{2} & -d
\end{array}\right)
$$

Then $\Omega X$ is an $N$-complex that is called the loop on $X$. For any $\omega \in \operatorname{Hom}_{\mathcal{C}_{N}(\mathscr{A})}(X, Y)$, define $\left(F_{\omega}\right)_{n}=(\Omega Y)_{n} \oplus X_{n}$ with differential given by

$$
d=\left(\begin{array}{cccccc}
0 & 1 & 0 & \cdots & 0 & 0 \\
\vdots & \vdots & \vdots & \vdots & \vdots & \vdots \\
0 & 0 & 0 & \cdots & 1 & 0 \\
-d^{N-1} & -d^{N-2} & -d^{N-3} & \cdots & -d & \omega \\
0 & 0 & 0 & \cdots & 0 & d
\end{array}\right)
$$

Then $F_{\omega}$ is an $N$-complex. We also have the following commutative diagram in $\mathcal{C}_{N}(\mathscr{A})$ with degreewise split exact rows:

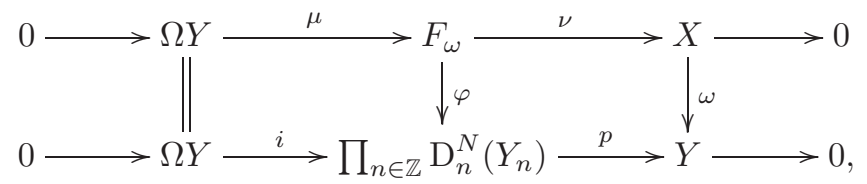

where $p=\left(d^{N-1}, d^{N-2}, \cdots, d, 1\right), \nu=(0, \cdots, 0,1)$, and

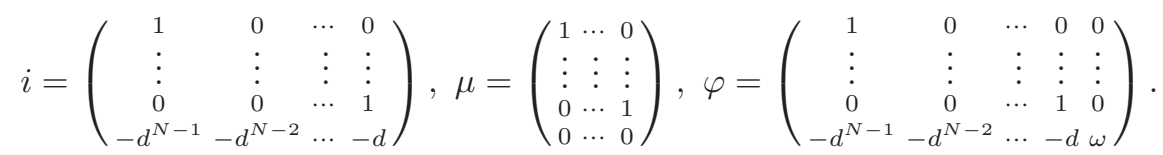

Define $(\Sigma X)_{n}=X_{n-1} \oplus X_{n-2} \oplus \cdots \oplus X_{n-(N-1)}$ with differential given by

$$
d=\left(\begin{array}{cccccc}
-d & 1 & 0 & \cdots & 0 & 0 \\
\vdots & \vdots & \vdots & \vdots & \vdots & \vdots \\
-d^{N-2} & 0 & 0 & \cdots & 0 & 1 \\
-d^{N-1} & 0 & 0 & \cdots & 0 & 0
\end{array}\right)
$$

Then $\Sigma X$ is an $N$-complex that is called the suspension on $X$. For any $\mu \in \operatorname{Hom}_{\mathcal{C}_{N}(\mathscr{A})}$ $(X, Y)$, define $\left(C_{\mu}\right)_{n}=Y_{n} \oplus(\Sigma X)_{n}$ with differential given by

$$
d=\left(\begin{array}{cccccc}
d & \mu & 0 & \cdots & 0 & 0 \\
0 & -d & 1 & \cdots & 0 & 0 \\
\vdots & \vdots & \vdots & \vdots & \vdots & \vdots \\
0 & -d^{N-2} & 0 & \cdots & 0 & 1 \\
0 & -d^{N-1} & 0 & \cdots & 0 & 0
\end{array}\right)
$$

Then $C_{\mu}$ is an $N$-complex. We also have the following commutative diagram in $\mathcal{C}_{N}(\mathscr{A})$ 
with degreewise split exact rows:

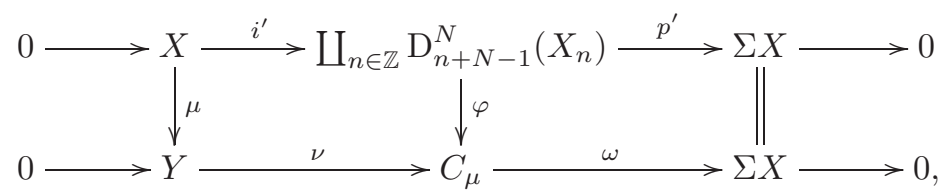

where

$$
\begin{gathered}
i^{\prime}=\left(\begin{array}{c}
1 \\
d \\
\vdots \\
d^{N-1}
\end{array}\right), p^{\prime}=\left(\begin{array}{cccccc}
-d & 1 & 0 & \cdots & 0 & 0 \\
-d^{2} & 0 & 1 & \cdots & 0 & 0 \\
\vdots & \vdots & \vdots & \vdots & \vdots & \vdots \\
-d^{N-1} & 0 & 0 & \cdots & 0 & 1
\end{array}\right), \\
\nu=\left(\begin{array}{c}
1 \\
0 \\
\vdots \\
0
\end{array}\right), \omega=\left(\begin{array}{ccccc}
0 & 1 & \cdots & 0 \\
\vdots & \vdots & \vdots & \vdots \\
0 & 0 & \cdots & 1
\end{array}\right), \varphi=\left(\begin{array}{ccccc}
\mu & 0 & 0 & \cdots & 0 \\
-d & 1 & 0 & \cdots & 0 \\
\vdots & \vdots & \vdots & \vdots & \vdots \\
-d^{N-1} & 0 & 0 & \cdots & 1
\end{array}\right) .
\end{gathered}
$$

We say a diagram in $\mathcal{K}_{N}(\mathscr{A})$ is a left triangle if it is isomorphic to a diagram

$$
\Omega Z \stackrel{\mu}{\longrightarrow} F_{\omega} \stackrel{\nu}{\longrightarrow} Y \stackrel{\omega}{\longrightarrow} Z
$$

arising from a chain map $\omega: Y \rightarrow Z$, where $\mu$ and $\nu$ are the canonical chain maps. We say a diagram in $\mathcal{K}_{N}(\mathscr{A})$ is a right triangle if it is isomorphic a diagram

$$
X \stackrel{\mu^{\prime}}{\longrightarrow} Y \stackrel{\nu^{\prime}}{\longrightarrow} C_{\mu^{\prime}} \stackrel{\omega^{\prime}}{\longrightarrow} \Sigma X
$$

arising from a chain map $\mu^{\prime}: X \rightarrow Y$, where $\nu^{\prime}$ and $\omega^{\prime}$ are the canonical chain maps.

If $N=2$, then $\Omega \Sigma X=X$ and $\Sigma \Omega X=X$ for any 2-complex $X$, i.e., $\Omega=\Sigma^{-1}$ and $\Sigma=\Omega^{-1}$. Moreover, the notion of left (or right) triangles coincides with the usual notion of distinguished triangles [13, p.11]. Murfet [13] proved that the additive category $\mathcal{K}_{2}(\mathscr{A})$ together with the additive automorphism $\Sigma$ and the class of distinguished triangles defined above is a triangulated category (see [13, Theorem 12]). But for the case of $N \geqslant 3$ there is no further information. However, Yang and Ding [16] proved the following result.

Theorem 2.2. ([16, Theorem 2.20]) The category $\mathcal{K}_{N}(\mathscr{A})$ together with the endofunctors $\Omega, \Sigma$ and the classes of left and right triangles defined above is a pretriangulated category.

Proposition 2.3. Let $\tau \in \operatorname{Hom}_{\mathcal{C}_{N}(\mathscr{A})}(X, Z)$ and let $\nu: F_{\tau} \rightarrow X, \nu^{\prime}: Z \rightarrow C_{\tau}$ be the canonical chain maps. Then:

(1) A chain map of $N$-complexes $f: C \rightarrow X$ factors through $\nu$ if and only if $\tau f \sim 0$.

(2) A chain map of $N$-complexes $g: Z \rightarrow C$ factors through $\nu^{\prime}$ if and only if $g \tau \sim 0$.

Proof. We just prove (1) since (2) is proved dually.

Suppose $\tau f \sim 0$. Then we have a commutative diagram of left triangles in $\mathcal{K}_{N}(\mathscr{A})$ :

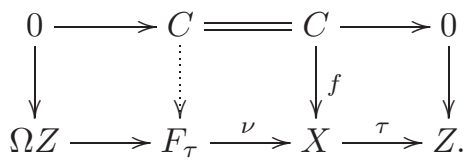

Therefore, (LT3) implies that $f$ factors through $\nu$. 
Suppose that $f: C \rightarrow X$ factors through $\nu$. Then we have the following commutative diagram of left triangles in $\mathcal{K}_{N}(\mathscr{A})$ :

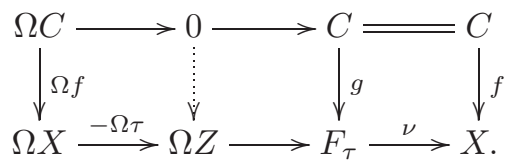

Therefore, (LT3) implies that $\Omega(\tau f) \sim 0$, and so $\tau f \sim 0$ by [16, Corollary 2.16].

Corollary 2.4. Let $f, g: X \rightarrow Z$ be chain maps of $N$-complexes. Then $f \sim g$ if and only if $f-g$ factors through the canonical chain map $F_{i d_{Z}} \rightarrow Z$ if and only if $f-$ $g$ factors through the canonical chain map $X \rightarrow C_{i d_{X}}$. In particular, we have the following exact sequences:

$$
\begin{aligned}
& \operatorname{Hom}_{\mathcal{C}_{N}(\mathscr{A})}\left(X, F_{i d_{Z}}\right) \rightarrow \operatorname{Hom}_{\mathcal{C}_{N}(\mathscr{A})}(X, Z) \rightarrow \operatorname{Hom}_{\mathcal{K}_{N}(\mathscr{A})}(X, Z) \rightarrow 0, \\
& \operatorname{Hom}_{\mathcal{C}_{N}(\mathscr{A})}\left(C_{i d_{X}}, Z\right) \rightarrow \operatorname{Hom}_{\mathcal{C}_{N}(\mathscr{A})}(X, Z) \rightarrow \operatorname{Hom}_{\mathcal{K}_{N}(\mathscr{A})}(X, Z) \rightarrow 0 .
\end{aligned}
$$

\section{Quasi-isomorphisms.}

A chain map $f: X \rightarrow Y$ induces homomorphisms $\mathrm{H}_{n}^{t}(f): \mathrm{H}_{n}^{t}(X) \rightarrow \mathrm{H}_{n}^{t}(Y)$ for all $n$ and $t$. We say that $f$ is a quasi-isomorphism if each $\mathrm{H}_{n}^{t}(f)$ is an isomorphism. Quasi-isomorphisms are marked by placing the sign $\simeq$ next to their arrow.

Lemma 2.5. ([16, Lemma 4.1]) The class $\mathscr{E}_{N}$ of exact $N$-complexes forms a thick subcategory of $\mathcal{K}_{N}(\mathscr{A})$. The corresponding class of morphisms

$$
\begin{aligned}
\operatorname{Mor}_{\mathscr{E}_{N}} & =\left\{\tau: X \rightarrow Z \mid \exists \text { a left triangle } \Omega Z \rightarrow E \rightarrow X \stackrel{\tau}{\rightarrow} Z \text { with } E \in \mathscr{E}_{N}\right\} \\
& =\left\{\tau: X \rightarrow Z \mid \exists \text { a right triangle } X \stackrel{\tau}{\rightarrow} Z \rightarrow E^{\prime} \rightarrow \Sigma X \text { with } E^{\prime} \in \mathscr{E}_{N}\right\}
\end{aligned}
$$

is the class of all quasi-isomorphisms in $\mathcal{K}_{N}(\mathscr{A})$.

The pretriangulated category $\mathcal{D}_{N}(\mathscr{A})$.

By [16, Lemma 4.3], $S=\operatorname{Mor}_{\mathscr{E}_{N}}$ is a saturated multiplicative system in $\mathcal{K}_{N}(\mathscr{A})$. Then one can define the quotient category $S^{-1} \mathcal{K}_{N}(\mathscr{A})=\mathcal{K}_{N}(\mathscr{A}) / \mathscr{E}_{N}$ of $\mathcal{K}_{N}(\mathscr{A})$ by $[\mathbf{7}, \S 8]$. The derived category $\mathcal{D}_{N}(\mathscr{A})$ of $\mathcal{C}_{N}(\mathscr{A})$ is the quotient category $\mathcal{K}_{N}(\mathscr{A}) / \mathscr{E}_{N}$. Then there exists a canonical functor

$$
Q: \mathcal{K}_{N}(\mathscr{A}) \longrightarrow \mathcal{D}_{N}(\mathscr{A})
$$

Let $\gamma: X \rightarrow Y$ be a morphism in $\mathcal{D}_{N}(\mathscr{A})$. By $[\mathbf{7}, \S 8]$, we can write $\gamma=Q(s)^{-1} Q(f)$ or $\gamma=Q(g) Q(t)^{-1}$ for some chain maps fitting into the diagrams of the forms

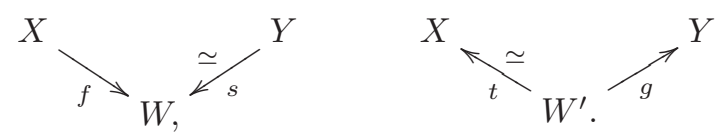

We say a diagram in $\mathcal{D}_{N}(\mathscr{A})$ is a left triangle if it is isomorphic to a diagram

$$
\Omega Z \stackrel{Q(\mu)}{\longrightarrow} X \stackrel{Q(\nu)}{\longrightarrow} Y \stackrel{Q(\omega)}{\longrightarrow} Z
$$


where $\Omega Z \stackrel{\mu}{\rightarrow} X \stackrel{\nu}{\rightarrow} Y \stackrel{\omega}{\rightarrow} Z$ is a left triangle in $\mathcal{K}_{N}(\mathscr{A})$. We say a diagram in $\mathcal{D}_{N}(\mathscr{A})$ is a right triangle if it is isomorphic to a diagram

$$
X^{\prime} \stackrel{Q\left(\mu^{\prime}\right)}{\longrightarrow} Y^{\prime} \stackrel{Q\left(\nu^{\prime}\right)}{\longrightarrow} Z^{\prime} \stackrel{Q\left(\omega^{\prime}\right)}{\longrightarrow} \Sigma X^{\prime}
$$

where $X^{\prime} \stackrel{\mu^{\prime}}{\rightarrow} Y^{\prime} \stackrel{\nu^{\prime}}{\rightarrow} Z^{\prime} \stackrel{\omega^{\prime}}{\rightarrow} \Sigma X^{\prime}$ is a right triangle in $\mathcal{K}_{N}(\mathscr{A})$. Then the category $\mathcal{D}_{2}(\mathscr{A})$ is a portly triangulated category (do not require the distinguished triangles to form a class) by $\left[\mathbf{7}\right.$, Proposition 9.8], and the category $\mathcal{D}_{N}(\mathscr{A})$ is a portly pretriangulated category whenever $N \geqslant 3$ by [16, Theorem 4.15].

\section{Homotopy resolutions of $N$-complexes}

The main aim of this section is to investigate the existence of $d g$-projective and $d g$-injective resolutions for arbitrary $N$-complexes.

Definition 3.1. Assume that $\mathscr{A}$ has enough projectives and let $\mathscr{P}$ be the class of projectives. An $N$-complex $P$ is called $d g$-projective if $P_{n} \in \mathscr{P}$ for all $n$, and every $f: P \rightarrow E$ is null homotopic whenever $E \in \mathscr{E}_{N}$. We denote the class of $d g$-projective $N$-complexes by $d g \widetilde{\mathscr{P}}_{N}$. Assume that $\mathscr{A}$ has enough injectives and let $\mathscr{I}$ be the class of injectives. An $N$-complex $I$ is called $d g$-injective if $I_{n} \in \mathscr{I}$ for all $n$, and every $f: E \rightarrow I$ is null homotopic whenever $E \in \mathscr{E}_{N}$. We denote the class of $d g$-injective $N$-complexes by $d g \widetilde{\mathscr{I}}_{N}$.

Lemma 3.2. ([16, Lemma 5.5]) (1) Assume $\mathscr{A}$ has enough projectives and let $\mathscr{P}$ be the class of projectives. Then every bounded-below $N$-complex of projectives is $d g$ projective.

(2) Assume $\mathscr{A}$ has enough injectives and let $\mathscr{I}$ be the class of injectives. Then every bounded-above $N$-complex of injectives is dg-injective.

Lemma 3.3. ([16, Lemma 5.2]) Let $X, Y$ be two $N$-complexes with either $X$ a dgprojective $N$-complex or $Y$ a dg-injective $N$-complex. Then we have an isomorphism

$$
\operatorname{Hom}_{\mathcal{K}_{N}(\mathscr{A})}(X, Y) \cong \operatorname{Hom}_{\mathcal{D}_{N}(\mathscr{A})}(X, Y)
$$

The first task is to construct $d g$-projective resolution of any bounded-below $N$ complex and $d g$-injective resolution of any bounded-above $N$-complex.

Proposition 3.4. Suppose $\mathscr{A}$ has enough projectives and let $\mathscr{P}$ be the class of projectives.

(1) Every bounded-below $N$-complex $X$ admits a quasi-isomorphism $P \stackrel{\simeq}{\rightrightarrows} X$ with $P$ a bounded-below $N$-complex in $\mathscr{P}$.

(2) If $f: X \rightarrow Y$ is a chain map of bounded-below $N$-complexes, $u: P \rightarrow X$ a quasi-isomorphism with $P$ a bounded-below $N$-complex in $\mathscr{P}$, then there is a unique, 
up to homotopy, chain map $g$ such that the following diagram in $\mathcal{K}_{N}(\mathscr{A})$ commutes:

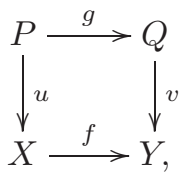

where $v: Q \rightarrow Y$ is a quasi-isomorphism and $Q$ a bounded-below $N$-complex in $\mathscr{P}$. In particular, if $f=i d_{X}$, then $g$ is a homotopy equivalence.

Proof. (1) Let $X$ be a bounded-below $N$-complex-say, $X_{i}=0$ for all $i<n$. For $i<n$ we define $P_{i}=0$. Choose any epimorphism $P_{n} \rightarrow X_{n}$ with $P_{n} \in \mathscr{P}$. Suppose that for some $k>n$ we have constructed an object $P_{k-1} \in \mathscr{P}$ and morphisms $d_{k-1}^{P}: P_{k-1} \rightarrow$ $P_{k-2}$ and $P_{k-1} \rightarrow X_{k-1}$. Consider the pullback diagram

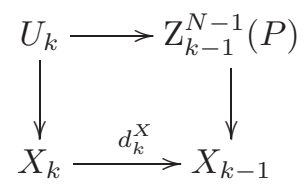

and choose an epimorphism $P_{k} \rightarrow U_{k}$ with $P_{k} \in \mathscr{P}$. Define morphisms $d_{k}^{P}: P_{k} \rightarrow$ $P_{k-1}$ and $P_{k} \rightarrow X_{k}$ in the obvious way. Consider the pullback diagram

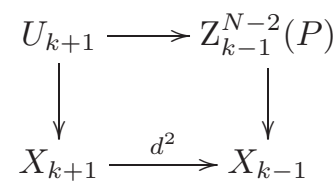

and choose an epimorphism $P_{k+1} \rightarrow U_{k+1}$ with $P_{k+1} \in \mathscr{P}$. Define morphisms $P_{k+1} \rightarrow$ $P_{k-1}$ and $P_{k+1} \rightarrow X_{k+1}$ in the obvious way. Applying the property of pullback for diagram (3.1), we get a commutative diagram:

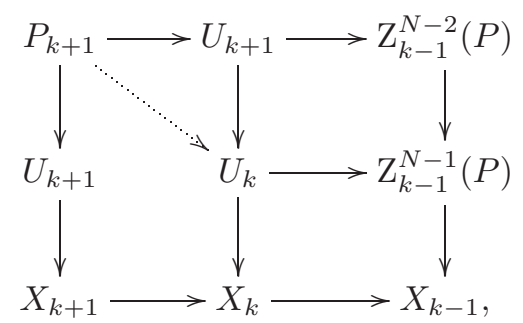

and hence there exists $d_{k+1}^{P}: P_{k+1} \rightarrow P_{k}$. Continuing this process, consider the pullback diagram

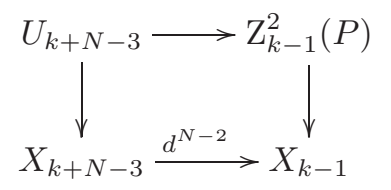

and choose an epimorphism $P_{k+N-3} \rightarrow U_{k+N-3}$ with $P_{k+N-3} \in \mathscr{P}$. Define morphisms $P_{k+N-3} \rightarrow P_{k-1}$ and $P_{k+N-3} \rightarrow X_{k+N-3}$ in the obvious way. Consider the 
pullback diagram

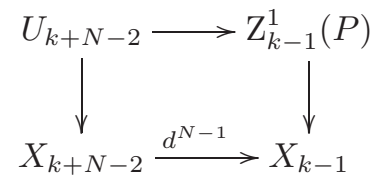

and choose an epimorphism $P_{k+N-2} \rightarrow U_{k+N-2}$ with $P_{k+N-2} \in \mathscr{P}$. Define morphisms $P_{k+N-2} \rightarrow P_{k-1}$ and $P_{k+N-2} \rightarrow X_{k+N-2}$ in the obvious way. Applying the property of pullback for diagram (3.3), we obtain a commutative diagram:

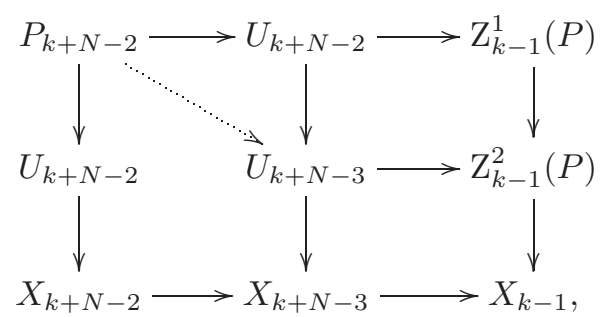

and so there exists $d_{k+N-2}^{P}: P_{k+N-2} \rightarrow P_{k+N-3}$. Repeating this process, we get an $N$-complex $P: \cdots \rightarrow P_{n+1} \rightarrow P_{n} \rightarrow 0 \rightarrow \cdots$ together with a chain map $P \rightarrow X$. It remains to show that $P \rightarrow X$ is a quasi-isomorphism. For $t=1, \cdots, N-1$, the above pullback diagrams give rise to a pullback diagram:

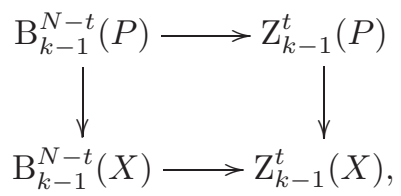

where the horizontal morphisms are monomorphisms. It follows from [13, Lemma 68] that the vertical morphism on the right is an epimorphism. Thus, [14, Lemma 35] implies that $\mathrm{H}_{k-1}^{t}(P) \cong \mathrm{H}_{k-1}^{t}(X)$, and hence $P \stackrel{\simeq}{\rightrightarrows} X$.

(2) Let $u: P \stackrel{\simeq}{\rightarrow} X$ and $v: Q \stackrel{\simeq}{\rightarrow} Y$ with $P, Q$ bounded-below $N$-complexes in $\mathscr{P}$. We complete $v$ to a left triangle $\Omega Y \rightarrow F_{v} \rightarrow Q \stackrel{v}{\rightarrow} Y$ and a right triangle $Q \stackrel{v}{\rightarrow} Y \rightarrow$ $C_{v} \rightarrow \Sigma Q$. Applying the functor $\operatorname{Hom}_{\mathcal{K}_{N}(\mathscr{A})}(P,-)$ for the above diagrams, we get exact sequences

$$
\begin{aligned}
& \operatorname{Hom}_{\mathcal{K}_{N}(\mathscr{A})}\left(P, F_{v}\right) \rightarrow \operatorname{Hom}_{\mathcal{K}_{N}(\mathscr{A})}(P, Q) \rightarrow \operatorname{Hom}_{\mathcal{K}_{N}(\mathscr{A})}(P, Y), \\
& \operatorname{Hom}_{\mathcal{K}_{N}(\mathscr{A})}(P, Q) \rightarrow \operatorname{Hom}_{\mathcal{K}_{N}(\mathscr{A})}(P, Y) \rightarrow \operatorname{Hom}_{\mathcal{K}_{N}(\mathscr{A})}\left(P, C_{v}\right) .
\end{aligned}
$$

Note that $C_{v}, F_{v}$ are exact and $P$ is bounded below; it follows from Lemma 3.2 that $0=\operatorname{Hom}_{\mathcal{K}_{N}(\mathscr{A})}\left(P, F_{v}\right)=\operatorname{Hom}_{\mathcal{K}_{N}(\mathscr{A})}\left(P, C_{v}\right)$. This implies that

$$
\operatorname{Hom}_{\mathcal{K}_{N}(\mathscr{A})}(P, Q) \cong \operatorname{Hom}_{\mathcal{K}_{N}(\mathscr{A})}(P, Y),
$$

and hence there is a unique, up to homotopy, chain map $g: P \rightarrow Q$ such that $v g \sim f u$. The second statement follows from Lemma 3.3.

The dual result is given by the following. 
Proposition 3.5. Suppose $\mathscr{A}$ has enough injectives and let $\mathscr{I}$ be the class of injectives.

(1) Every bounded-above $N$-complex $X$ admits a quasi-isomorphism $X \stackrel{\simeq}{\rightarrow} I$ with $I$ a bounded-above $N$-complex in $\mathscr{I}$.

(2) If $f: Y \rightarrow X$ is a chain map of bounded-above $N$-complexes, $u: X \rightarrow I$ a quasiisomorphism with $I$ a bounded-above $N$-complex in $\mathscr{I}$, then there is a unique, up to homotopy, chain map g such that the following diagram in $\mathcal{K}_{N}(\mathscr{A})$ commutes:

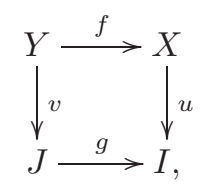

where $v: Y \rightarrow J$ is a quasi-isomorphism and $J$ a bounded-above $N$-complex in $\mathscr{I}$. In particular, if $f=i d_{X}$, then $g$ is a homotopy equivalence.

We now investigate the existence of $d g$-projective resolution and $d g$-injective resolution for arbitrary $N$-complexes.

Let $X$ be an $N$-complex and $n \in \mathbb{Z}$. We define $X_{\supset n}$ to be the $N$-complex

$$
X_{\supset n}: \cdots \longrightarrow X_{n+2} \stackrel{d}{\longrightarrow} X_{n+1} \stackrel{d}{\longrightarrow} \mathrm{Z}_{n}^{N-1}(X) \longrightarrow 0 .
$$

There exists an obvious monomorphism of $N$-complexes $u: X_{\supset_{n}} \longmapsto X$. As subobjects of $X$, it is clear that $X_{\supset n} \longmapsto X_{\supset n-1}$. In fact, the inclusions $\left\{X_{\supset n} \longmapsto X\right\}_{n \leqslant 0}$ are a direct limit in $\mathcal{C}_{N}(\mathscr{A})$. That is, $X=\lim _{n \leqslant 0} X_{\supset n}$. We also define $X_{\subset n}$ to be the $N$ complex

$$
X_{\subset n}: 0 \longrightarrow \mathrm{C}_{n}^{N-1}(X) \stackrel{\bar{d}}{\longrightarrow} X_{n-1} \stackrel{d}{\longrightarrow} X_{n-2} \stackrel{d}{\longrightarrow} \cdots,
$$

where the differential $\bar{d}$ is the induced morphism on residue classes. There is an obvious epimorphism of $N$-complexes $X \rightarrow X_{\subset n}$. As quotients of $X$, it is clear that $X_{\subset n} \rightarrow X_{\subset n-1}$. In fact, the quotients $\left\{X \rightarrow X_{\subset n}\right\}_{n \geqslant 0}$ are an inverse limit in $\mathcal{C}_{N}(\mathscr{A})$. That is, $X=\lim _{n \geqslant 0} X_{\subset n}$.

Remark 3.6. The set $\mathbb{N}=\{0,1,2, \cdots\}$ is a directed set in the canonical way (with minimum 0). Let $\mathscr{A}$ be a cocomplete abelian category, and suppose we are given a direct system $\left\{G^{n}, u^{n m}\right\}_{n \in \mathbb{N}}$ over this directed set. This is just a sequence of objects and morphisms in $\mathscr{A}$ (writing $u^{n}$ for $u^{n(n+1)}$ )

$$
G^{0} \stackrel{u^{0}}{\longrightarrow} G^{1} \stackrel{u^{1}}{\longrightarrow} G^{2} \stackrel{u^{2}}{\longrightarrow} G^{3} \longrightarrow \cdots .
$$

Let $v: \coprod_{n \in \mathbb{N}} G^{n} \rightarrow \coprod_{n \in \mathbb{N}} G^{n}$ be the morphism induced out of the first coproduct by the morphisms $u^{n}: G^{n} \rightarrow G^{n+1}$. That is, $v \iota^{n}=\iota^{n+1} u^{n}$ where $\iota^{n}$ is the injection of $G^{n}$ into the coproduct. Given a cokernel $\coprod_{n \in \mathbb{N}} G^{n} \rightarrow C$ of the morphism $1-v$ it is clear that the composites $G^{n} \rightarrow \coprod_{n \in \mathbb{N}} G^{n} \rightarrow C$ are a direct limit for direct system $\left\{G^{n}, u^{n m}\right\}_{n \in \mathbb{N}}$. 
Definition 3.7. Let $\mathscr{A}$ be a cocomplete abelian category, and suppose we have the following sequence of chain maps of $N$-complexes

$$
G^{0} \stackrel{u^{0}}{\longrightarrow} G^{1} \stackrel{u^{1}}{\longrightarrow} G^{2} \stackrel{u^{2}}{\longrightarrow} G^{3} \longrightarrow \cdots .
$$

Then $\mathcal{C}_{N}(\mathscr{A})$ is a cocomplete abelian category, so as in Remark 3.6 we can define a chain map $v: \coprod_{n \in \mathbb{N}} G^{n} \rightarrow \coprod_{n \in \mathbb{N}} G^{n}$. The homotopy direct limit of the above sequence is the suspension $C_{1-v}$ on $\coprod_{n \in \mathbb{N}} G^{n}$, and we denote the object $C_{1-v}$ by $\underline{h o l i m} G^{n}$. This means that there exists a right triangle in $\mathcal{K}_{N}(\mathscr{A})$ :

$$
\coprod_{n \in \mathbb{N}} G^{n} \stackrel{1-v}{\longrightarrow} \coprod_{n \in \mathbb{N}} G^{n} \longrightarrow \underline{\text { holim }} G^{n} \longrightarrow \Sigma\left(\coprod_{n \in \mathbb{N}} G^{n}\right) .
$$

Given a morphism of direct systems of $N$-complexes,

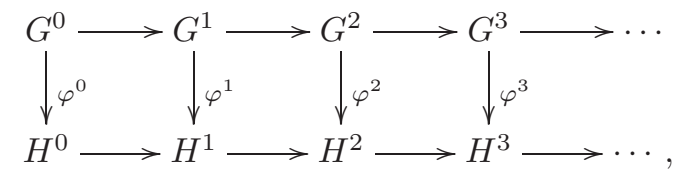

(RT3) gives a chain map $\underset{\text { holim }}{\longrightarrow} \varphi^{n}$ making the diagram

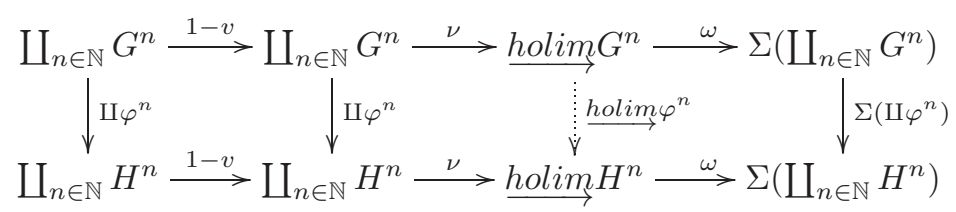

in $\mathcal{K}_{N}(\mathscr{A})$ commutative, where $\nu$ and $\omega$ are the canonical chain maps.

Theorem 3.8. Suppose $\mathscr{A}$ is a Grothendieck category with enough projectives, and let $\mathscr{P}$ be the class of projectives. Then every $N$-complex $X$ admits a quasi-isomorphism $P \stackrel{\simeq}{\rightrightarrows} X$ with $P$ a dg-projective $N$-complex.

Proof. Let $X$ be any $N$-complex. Since the $N$-complex $X_{\supset n}$ is bounded below for $n \leqslant 0$, we can find a bounded-below $N$-complex $P^{n}$ in $\mathscr{P}$ and a quasi-isomorphism $\theta^{n}: P^{n} \stackrel{\simeq}{\rightrightarrows} X_{\supset n}$ by Proposition 3.4(1). Using Proposition 3.4(2) at each stage, we can choose these resolutions in such a way that we have the following commutative diagram of $N$-complexes:

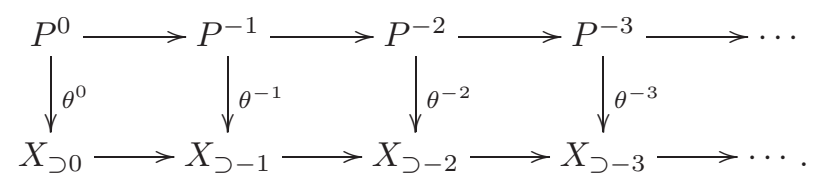

Thus there is an induced morphism of right triangles in $\mathcal{K}_{N}(\mathscr{A})$ :

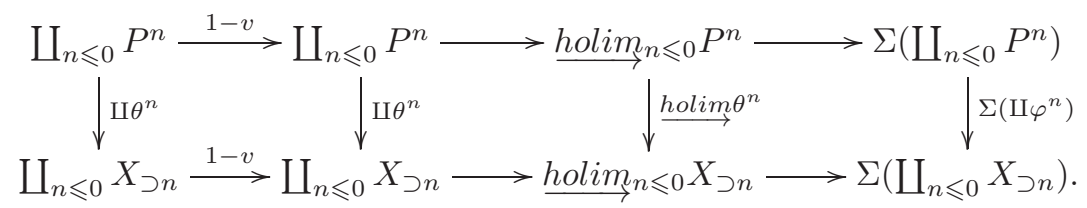


Applying canonical functor $Q$ to the above diagram, we can obtain a morphism of right triangles in $\mathcal{D}_{N}(\mathscr{A})$. But $Q\left(\amalg \theta^{n}\right)$ is an isomorphism; it follows that $Q\left(\underline{\text { holim }} \theta^{n}\right)$ is an isomorphism. Thus [16, Lemma 4.3] and [7, Corollary 8.9] imply that holim $\theta^{n}$ is a quasi-isomorphism. On the other hand, $1-v$ is a monomorphism by assumption and [13, Lemma 63]. Therefore, [16, Theorem 4.16] and (RT3) give a quasiisomorphism holim ${ }_{n \leqslant 0} X_{\supset n} \rightarrow \lim _{n \leqslant 0} X_{\supset n}=X$. Composing with holim $\theta^{n}$ yields a quasi-isomorphism $\underline{\text { holim }}_{n \leqslant 0} P^{n}=P \rightarrow X$. Note that

$$
\begin{gathered}
\operatorname{Hom}_{\mathcal{K}_{N}(\mathscr{A})}\left(\Sigma\left(\coprod_{n \leqslant 0} P^{n}\right), E\right) \rightarrow \operatorname{Hom}_{\mathcal{K}_{N}(\mathscr{A})}\left(\text { holim }_{n \leqslant 0} P^{n}, E\right) \rightarrow \\
\operatorname{Hom}_{\mathcal{K}_{N}(\mathscr{A})}\left(\coprod_{n \leqslant 0} P^{n}, E\right)
\end{gathered}
$$

is exact and $\coprod_{n \leqslant 0} P^{n}$ is $d g$-projective; it follows from [16, Lemma 2.17] that

$$
\operatorname{Hom}_{\mathcal{K}_{N}(\mathscr{A})}\left(\Sigma\left(\coprod_{n \leqslant 0} P^{n}\right), E\right) \cong \operatorname{Hom}_{\mathcal{K}_{N}(\mathscr{A})}\left(\coprod_{n \leqslant 0} P^{n}, \Omega E\right)=0, \forall E \in \mathscr{E}_{N} .
$$

This implies that $P$ is a $d g$-projective $N$-complex.

Remark 3.9. The set $\mathbb{N}^{o p}=\{0,1,2, \cdots\}$ is an inverse directed set in the canonical way (with maximum 0 ). Let $\mathscr{A}$ be a complete abelian category, and suppose we are given an inverse system $\left\{G^{n}, u^{n m}\right\}_{n \in \mathbb{N}}$ over this inverse directed set. This is just a sequence of objects and morphisms in $\mathscr{A}$ (writing $u^{n}$ for $u^{n(n-1)}$ )

$$
\cdots \longrightarrow G^{3} \stackrel{\mu^{3}}{\longrightarrow} G^{2} \stackrel{\mu^{2}}{\longrightarrow} G^{1} \stackrel{\mu^{1}}{\longrightarrow} G^{0} .
$$

Let $v: \prod_{n \in \mathbb{N}} G^{n} \rightarrow \prod_{n \in \mathbb{N}} G^{n}$ be the morphism induced into the second product by the morphisms $u^{n+1}: G^{n+1} \rightarrow G^{n}$. That is, $\pi^{n} v=u^{n+1} \pi^{n+1}$ where $\pi^{n}$ is the projection onto of $G^{n}$ out of the product. Given a kernel $K \rightarrow \prod_{n \in \mathbb{N}} G^{n}$ of the morphism $1-v$ it is clear that the composites $K \rightarrow \prod_{n \in \mathbb{N}} G^{n} \rightarrow G^{n}$ are an inverse limit for inverse system $\left\{G^{n}, u^{n m}\right\}_{n \in \mathbb{N}}$.

Definition 3.10. Let $\mathscr{A}$ be a complete abelian category, and suppose we have the following sequence of chain maps of $N$-complexes

$$
\cdots \longrightarrow G^{3} \stackrel{u^{3}}{\longrightarrow} G^{2} \stackrel{u^{2}}{\longrightarrow} G^{1} \stackrel{u^{1}}{\longrightarrow} G^{0} .
$$

Then $\mathcal{C}_{N}(\mathscr{A})$ is a complete abelian category, so as in Remark 3.9 we can define a chain map $v: \prod_{n \in \mathbb{N}} G^{n} \rightarrow \prod_{n \in \mathbb{N}} G^{n}$. The homotopy inverse limit of the above sequence is the loop $F_{1-v}$ on $\prod_{n \in \mathbb{N}} G^{n}$, and we denote the object $F_{1-v}$ by holim $G^{n}$. This means that there exists a left triangle in $\mathcal{K}_{N}(\mathscr{A})$ :

$$
\Omega\left(\prod_{n \in \mathbb{N}} G^{n}\right) \longrightarrow \text { holim }^{n} \longrightarrow \prod_{n \in \mathbb{N}} G^{n} \stackrel{1-\nu}{\longrightarrow} \prod_{n \in \mathbb{N}} G^{n} .
$$

Given a morphism of inverse systems of $N$-complexes:

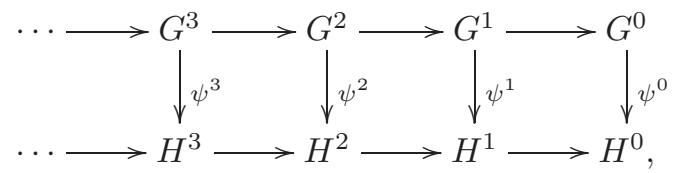


(LT3) gives a chain map holim $\psi^{n}$ making the diagram

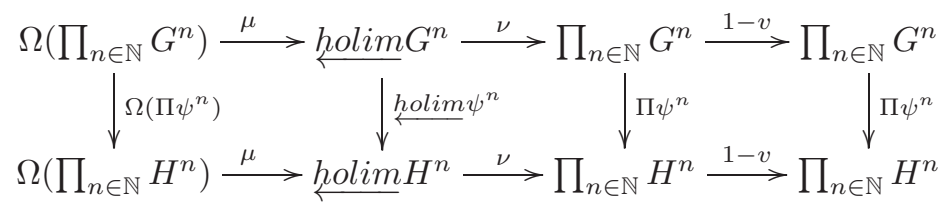

in $\mathcal{K}_{N}(\mathscr{A})$ commutative, where $\mu$ and $\nu$ are the canonical chain maps.

Theorem 3.11. Suppose $\mathscr{A}$ is a Grothendieck category with enough projectives, and let $\mathscr{I}$ be the class of injectives. Then every $N$-complex $X$ admits a quasi-isomorphism $X \stackrel{\sim}{\rightarrow} I$ with $I$ a dg-injective $N$-complex.

Proof. The proof is dual to that of Theorem 3.8.

\section{The homotopy categories $\mathcal{D}_{N}(R)$ and $\mathcal{K}_{N}(\mathscr{I})$}

In this section, $\mathscr{A}$ is the category of left $R$-modules and $\mathscr{I}$ the class of injectives. We denote the homotopy category of $N$-complexes in $\mathscr{I}$ by $\mathcal{K}_{N}(\mathscr{I})$. We show that the category $\mathcal{D}_{N}(R)$ is compactly generated, and the category $\mathcal{K}_{N}(\mathscr{I})$ is compactly generated when $R$ is left noetherian.

Definition 4.1. Let $\mathscr{T}$ be a pretriangulated category closed under set-indexed coproducts. An object $C \in \mathscr{T}$ is compact if the natural map

$$
\amalg_{\lambda \in \Lambda} \operatorname{Hom}_{\mathscr{T}}\left(C, X_{\lambda}\right) \longrightarrow \operatorname{Hom}_{\mathscr{T}}\left(C, \coprod_{\lambda \in \Lambda} X_{\lambda}\right)
$$

is an isomorphism for any family $\left\{X_{\lambda}\right\}_{\lambda \in \Lambda}$ of objects in $\mathscr{T}$. We denote by $\mathscr{T}^{c}$ the full subcategory of $\mathscr{T}$ that is formed by all compact objects. Clearly, $\mathscr{T}^{c}$ is right triangulated. A set of objects $\mathscr{G} \subseteq \mathscr{T}$ is called a generating set if the implication

$$
\operatorname{Hom}_{\mathscr{T}}(G, X)=0 \text { for all } G \in \mathscr{G} \Longrightarrow X=0
$$

holds for all objects $X \in \mathscr{T}$. If $\mathscr{T}$ has a generating set consisting of compact objects, then $\mathscr{T}$ is called compactly generated.

Lemma 4.2. For a morphism $u: X \rightarrow Y$ in $\mathcal{K}_{N}(R), Q(u)=0$ in $\mathcal{D}_{N}(R)$ if and only if $u$ factors through an exact $N$-complex.

Proof. "Only if" part. By [7, Proposition 8.19] there is a quasi-isomorphism $s: Z \rightarrow$ $X$ such that $u s=0$. Hence (RT3) yields a morphism of right triangles:

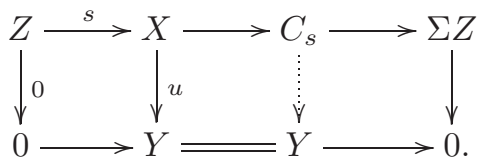

Note that $C_{s}$ is exact by [16, Corollary 3.3], so the proof is complete.

"If" part. Assume $u$ factors through $Z \in \mathscr{E}_{N}$. Then $Q(u)$ factors through $Q(Z)=0$, which implies that $Q(u)=0$ in $\mathcal{D}_{N}(R)$. 
Lemma 4.3. The canonical functor $Q: \mathcal{K}_{N}(R) \rightarrow \mathcal{D}_{N}(R)$ preserves direct sums. In particular, $\mathcal{D}_{N}(R)$ has arbitrary direct sums, which are direct sums of $N$-complexes.

Proof. Let $\left\{X_{\lambda}\right\}_{\lambda \in \Lambda}$ be a family of objects in $\mathcal{C}_{N}(R)$, and let $Y$ be an object in $\mathcal{C}_{N}(R)$. By Corollary 2.4 we have a commutative diagram with exact rows:

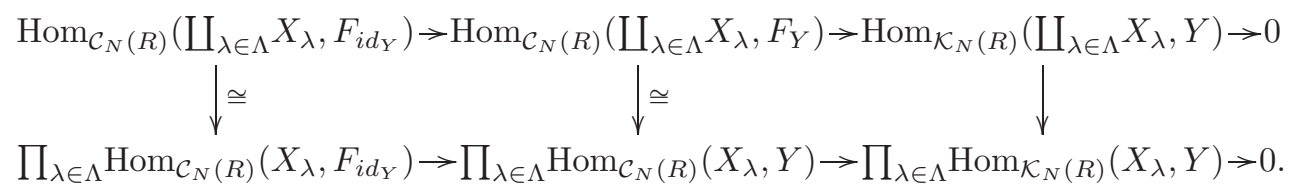

Thus the canonical homomorphism $\operatorname{Hom}_{\mathcal{K}_{N}(R)}\left(\coprod_{\lambda \in \Lambda} X_{\lambda}, Y\right) \rightarrow \prod_{\lambda \in \Lambda} \operatorname{Hom}_{\mathcal{K}_{N}(R)}$ $\left(X_{\lambda}, Y\right)$ is an isomorphism. This implies that arbitrary direct sums exist in $\mathcal{K}_{N}(R)$.

Let $\left\{X_{\lambda}\right\}_{\lambda \in \Lambda}$ be a family of objects in $\mathcal{K}_{N}(R)$. For each $\mu \in \Lambda$, we denote by $\iota_{\mu}: X_{\mu} \rightarrow \coprod_{\lambda \in \Lambda} X_{\lambda}$ the injection. We claim that the canonical homomorphism

$$
\xi_{Y}: \operatorname{Hom}_{\mathcal{D}_{N}(R)}\left(\coprod_{\lambda \in \Lambda} X_{\lambda}, Y\right) \rightarrow \prod_{\lambda \in \Lambda} \operatorname{Hom}_{\mathcal{D}_{N}(R)}\left(X_{\lambda}, Y\right), \gamma \mapsto\left(\gamma Q\left(\iota_{\mu}\right)\right)
$$

is an isomorphism for any object $Y$ in $\mathcal{D}_{N}(R)$.

Claim 1: $\xi_{Y}$ is an epimorphism.

Proof. Let $\left(\gamma_{\lambda}\right)_{\lambda \in \Lambda} \in \prod_{\lambda \in \Lambda} \operatorname{Hom}_{\mathcal{D}_{N}(R)}\left(X_{\lambda}, Y\right)$. For each $\mu \in \Lambda$, let $\gamma_{\mu}=$ $Q\left(f_{\mu}\right) Q\left(s_{\mu}\right)^{-1}$ with $s_{\mu}: W_{\mu} \rightarrow X_{\mu}$ a quasi-isomorphism and embed $s_{\mu}$ in a right triangle $W_{\mu} \stackrel{s_{\mu}}{\rightarrow} X_{\mu} \rightarrow C_{\mu} \rightarrow \Sigma W_{\mu}$ in $\mathcal{K}_{N}(R)$. We show that $\coprod_{\lambda \in \Lambda} W_{\lambda} \stackrel{\amalg_{\lambda \in \Lambda}{ }^{s_{\lambda}}}{{ }^{\prime}} \coprod_{\lambda \in \Lambda} X_{\lambda} \rightarrow$ $\coprod_{\lambda \in \Lambda} C_{\lambda} \rightarrow \Sigma \coprod_{\lambda \in \Lambda} W_{\lambda}$ is a right triangle in $\mathcal{K}_{N}(R)$. Note first that there exists a natural isomorphism $\Sigma \coprod_{\lambda \in \Lambda} W_{\lambda} \cong \coprod_{\lambda \in \Lambda} \Sigma W_{\lambda}$. By (RT1) we have a right triangle $\coprod_{\lambda \in \Lambda} W_{\lambda} \stackrel{\amalg_{\lambda \in \Lambda}{ }^{s}}{\rightarrow} \coprod_{\lambda \in \Lambda} X_{\lambda} \rightarrow C \rightarrow \Sigma \coprod_{\lambda \in \Lambda} W_{\lambda}$ in $\mathcal{K}_{N}(R)$. Then for each $\mu \in \Lambda$, we have a morphism of right triangles:

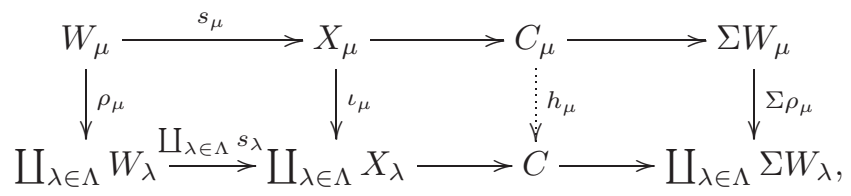

where $\rho_{\mu}: W_{\mu} \rightarrow \coprod_{\lambda \in \Lambda} W_{\lambda}$ is the injective. Thus we get a commutative diagram:

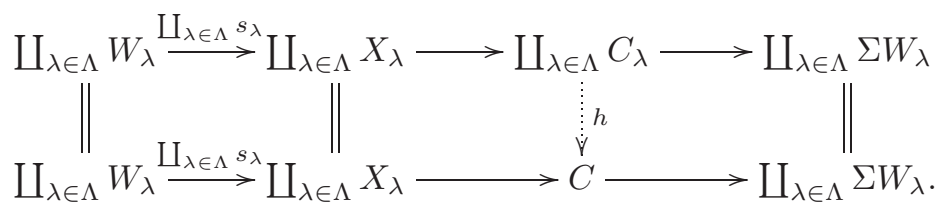


Next we show that $h$ is an isomorphism. We have a commutative diagram of functors:

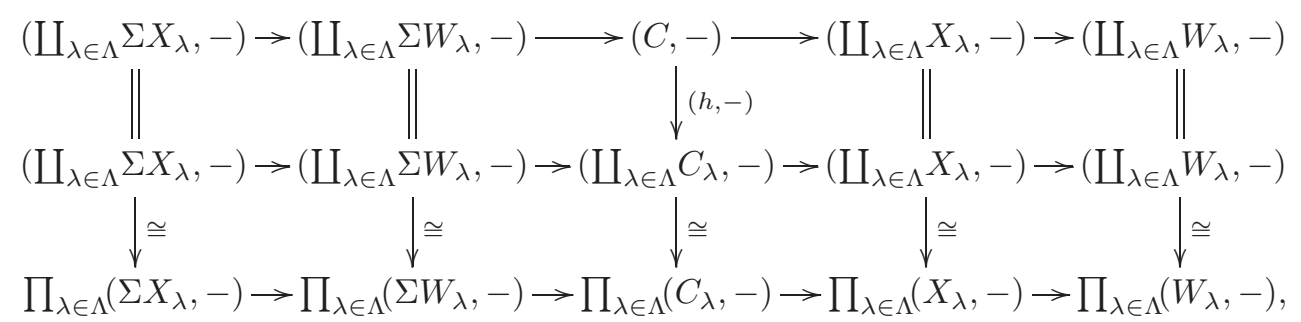

where $(-,-)=\operatorname{Hom}_{\mathcal{K}_{N}(R)}(-,-)$. Note that the top and the bottom rows are exact, as is the middle one. Thus by the five lemma, $(h,-)$ is an isomorphism, and so is $h$ by Yoneda lemma. Also, since $C_{\lambda}$ is exact for all $\lambda \in \Lambda, \coprod_{\lambda \in \Lambda} C_{\lambda}$ is exact and so $\coprod_{\lambda \in \Lambda} s_{\lambda}$ is a quasi-isomorphism by [16, Corollary 3.3]. For each $\mu \in \Lambda$, there exists $f \in \operatorname{Hom}_{\mathcal{K}_{N}(R)}\left(\amalg_{\lambda \in \Lambda} W_{\lambda}, Y\right)$ such that $f_{\mu}=f \rho_{\mu}$. Set $\gamma=Q(f) Q\left(\amalg_{\lambda \in \Lambda} s_{\lambda}\right)^{-1}$. Then, for all $\mu \in \Lambda$, we have

$$
\gamma Q\left(\iota_{\mu}\right)=Q(f) Q\left(\coprod_{\lambda \in \Lambda} s_{\lambda}\right)^{-1} Q\left(\iota_{\mu}\right)=Q(f) Q\left(\rho_{\mu}\right) Q\left(s_{\mu}\right)^{-1}=Q\left(f_{\mu}\right) Q\left(s_{\mu}\right)^{-1}=\gamma_{\mu} .
$$

Claim 2: $\xi_{Y}$ is a monomorphism.

Proof. Let $\gamma \in \operatorname{Hom}_{\mathcal{D}_{N}(R)}\left(\amalg_{\lambda \in \Lambda} X_{\lambda}, Y\right)$ with $\gamma Q\left(\iota_{\lambda}\right)=0$ for all $\lambda \in \Lambda$, and let $\gamma=Q(t)^{-1} Q(g)$ with $t: Y \rightarrow Y^{\prime}$ a quasi-isomorphism. We claim that $Q(g)=0$. For any $\lambda \in \Lambda$, since $Q(t)^{-1} Q\left(g \iota_{\lambda}\right)=0, Q\left(g \iota_{\lambda}\right)=0$. Then Lemma 4.2 implies that $g \iota_{\lambda}$ factors through some $E_{\lambda} \in \mathscr{E}_{N}$, and so $g$ factors through $\coprod_{\lambda \in \Lambda} E_{\lambda} \in \mathscr{E}_{N}$. Therefore, again by Lemma $4.2, Q(g)=0$.

Lemma 4.4. For any $R$-module $A$ and any $N$-complex $X$, we have isomorphisms

$$
\begin{gathered}
\operatorname{Hom}_{\mathcal{K}_{N}(R)}\left(\mathrm{D}_{n}^{t}(A), X\right) \cong \mathrm{H}_{n}^{t}\left(\operatorname{Hom}_{R}(A, X)\right), \forall n, t, \\
\operatorname{Hom}_{\mathcal{K}_{N}(R)}\left(X, \mathrm{D}_{n+t-1}^{t}(A)\right) \cong \mathrm{H}_{n}^{N-t}\left(\operatorname{Hom}_{R}(X, A)\right), \forall n, t .
\end{gathered}
$$

Proof. We just prove the first isomorphism since the second follows by duality.

We may consider that

$$
\begin{aligned}
\operatorname{Hom}_{\mathcal{C}_{N}(R)}\left(\mathrm{D}_{n}^{t}(A), X\right) & \cong \operatorname{Hom}_{R}\left(A, \mathrm{Z}_{n}^{t}(X)\right) \\
& =\left\{u \in \operatorname{Hom}_{R}\left(A, X_{n}\right) \mid d^{t} u=0\right\} \\
& =\mathrm{Z}_{n}^{t}\left(\operatorname{Hom}_{R}(A, X)\right),
\end{aligned}
$$

$$
\operatorname{Hom}_{\mathcal{C}_{N}(R)}\left(\mathrm{D}_{n+N-t}^{N}(A), X\right)=\left\{(v, u) \in \operatorname{Hom}_{R}\left(A, X_{n+N-t}\right) \times \operatorname{Hom}_{R}\left(A, X_{n}\right) \mid u=\right.
$$

$$
\left.d^{N-t} v\right\} \text {. }
$$

For $i: \mathrm{D}_{n}^{t}(A) \rightarrow \mathrm{D}_{n+N-t}^{N}(A)$, we get $\operatorname{Im}\left(\operatorname{Hom}_{\mathcal{C}_{N}(R)}(i, X)\right)=\mathrm{B}_{n}^{N-t}\left(\operatorname{Hom}_{R}(A, X)\right)$. Let $f: \mathrm{D}_{n}^{t}(A) \rightarrow X$ be a chain map. If $f \in \operatorname{Im}\left(\operatorname{Hom}_{\mathcal{C}_{N}(R)}(i, X)\right)$, then $f \sim 0$. Conversely, if $f \sim 0$, then $f$ factors through the $N$-complex $\coprod_{n \in \mathbb{Z}} \mathrm{D}_{n}^{N}\left(X_{n}\right)$ by the proof of $[\mathbf{5}$, Corollary 3.5], which induces a chain map $\mathrm{D}_{n+N-t}^{N}(A) \rightarrow X$. So $f \in \operatorname{Im}\left(\operatorname{Hom}_{\mathcal{C}_{N}(R)}(i, X)\right)$ and

$$
\operatorname{Hom}_{\mathcal{C}_{N}(R)}\left(\mathrm{D}_{n+N-t}^{N}(A), X\right) \rightarrow \operatorname{Hom}_{\mathcal{C}_{N}(R)}\left(\mathrm{D}_{n}^{t}(A), X\right) \rightarrow \operatorname{Hom}_{\mathcal{K}_{N}(R)}\left(\mathrm{D}_{n}^{t}(A), X\right) \rightarrow 0
$$

is exact; it follows that $\operatorname{Hom}_{\mathcal{K}_{N}(R)}\left(\mathrm{D}_{n}^{t}(A), X\right) \cong \mathrm{H}_{n}^{t}\left(\operatorname{Hom}_{R}(A, X)\right)$. 
Theorem 4.5. The pretriangulated category $\mathcal{D}_{N}(R)$ is compactly generated.

Proof. Set $\mathscr{G}=\left\{\mathrm{D}_{n}^{t}(R) \mid n \in \mathbb{Z}, t=1, \cdots, N-1\right\}$. We show that $\mathscr{G}$ is a generating set of $\mathcal{D}_{N}(R)$. First, for any $N$-complex $X$, assume that $\operatorname{Hom}_{\mathcal{D}_{N}(R)}\left(\mathrm{D}_{n}^{t}(R), X\right)=0$ for all $n$ and $t$. Then $0=\operatorname{Hom}_{\mathcal{D}_{N}(R)}\left(\mathrm{D}_{n}^{t}(R), X\right) \cong \operatorname{Hom}_{\mathcal{K}_{N}(R)}\left(\mathrm{D}_{n}^{t}(R), X\right) \cong \mathrm{H}_{n}^{t}(X)$ by Lemmas 3.3 and 4.4 since $\mathrm{D}_{n}^{t}(R)$ is $d g$-projective for all $n$ and $t$. This implies that $X \cong 0$ in $\mathcal{D}_{N}(R)$. Now let $\left\{X_{\lambda}\right\}_{\lambda \in \Lambda}$ be family of objects in $\mathcal{D}_{N}(R)$. Then the direct sum $\bigsqcup_{\lambda \in \Lambda} X_{\lambda}$ exists in $\mathcal{D}_{N}(R)$ by Lemma 4.3 and we have

$$
\begin{aligned}
\operatorname{Hom}_{\mathcal{D}_{N}(R)}\left(\mathrm{D}_{n}^{t}(R), \coprod_{\lambda \in \Lambda} X_{\lambda}\right) & \cong \operatorname{Hom}_{\mathcal{K}_{N}(R)}\left(\mathrm{D}_{n}^{t}(R), \coprod_{\lambda \in \Lambda} X_{\lambda}\right) \\
& \cong \mathrm{H}_{n}^{t}\left(\coprod_{\lambda \in \Lambda} X_{\lambda}\right) \cong \coprod_{\lambda \in \Lambda} \mathrm{H}_{n}^{t}\left(X_{\lambda}\right) \\
& \cong \coprod_{\lambda \in \Lambda} \operatorname{Hom}_{\mathcal{K}_{N}(R)}\left(\mathrm{D}_{n}^{t}(R), X_{\lambda}\right) \\
& \cong \coprod_{\lambda \in \Lambda} \operatorname{Hom}_{\mathcal{D}_{N}(R)}\left(\mathrm{D}_{n}^{t}(R), X_{\lambda}\right) .
\end{aligned}
$$

Therefore, $\mathrm{D}_{n}^{t}(R)$ is a compact object in $\mathcal{D}_{N}(R)$ for all $n$ and $t$. This implies that the pretriangulated category $\mathcal{D}_{N}(R)$ is compactly generated.

Now we show that the category $\mathcal{K}_{N}(\mathscr{I})$ is compactly generated whenever $R$ is left noetherian. For $N=2$ this was proved by Krause in [12, Proposition 2.3].

Remark 4.6. Let $A$ be a left $R$-module. Then Proposition 3.5(1) gives a quasiisomorphism $\mathrm{D}_{n}^{t}(A) \stackrel{\simeq}{\rightrightarrows} I$ with $I$ a bounded-above $N$-complex in $\mathscr{I}$ for any $n$ and $t$. We denote the $N$-complex $I$ by $i_{n}^{t} A$.

Let $X$ be an $N$-complex and $n \in \mathbb{Z}$. We define $X_{\leqslant n}$ to be the $N$-complex

$$
X_{\leqslant n}: 0 \longrightarrow X_{n} \stackrel{d}{\longrightarrow} X_{n-1} \stackrel{d}{\longrightarrow} X_{n-2} \longrightarrow \cdots .
$$

We also define $X_{\geqslant n}$ to be the $N$-complex

$$
X_{\geqslant n}: \cdots \longrightarrow X_{n+2} \stackrel{d}{\longrightarrow} X_{n+1} \stackrel{d}{\longrightarrow} X_{n} \longrightarrow 0 .
$$

Lemma 4.7. Let $R$ be left noetherian and $A$ a left $R$-module. Then the natural map

$$
\operatorname{Hom}_{\mathcal{K}_{N}(R)}\left(i_{n}^{t} A, X\right) \longrightarrow \operatorname{Hom}_{\mathcal{K}_{N}(R)}\left(D_{n}^{t}(A), X\right)
$$

is an isomorphism for all $X \in \mathcal{K}_{N}(\mathscr{I})$ and all $n, t$. Therefore, $i_{n}^{t} A$ is a compact object in $\mathcal{K}_{N}(\mathscr{I})$ for all $n$ and $t$ whenever $A$ is finitely generated.

Proof. We can complete the quasi-isomorphism $\mathrm{D}_{n}^{t}(A) \stackrel{\iota}{\rightarrow} i_{n}^{t} A$ to a right triangle $\mathrm{D}_{n}^{t}(A) \stackrel{\iota}{\rightarrow} i_{n}^{t} A \rightarrow C_{\iota} \rightarrow \Sigma\left(\mathrm{D}_{n}^{t}(A)\right)$ and a left triangle $\Omega\left(i_{n}^{t} A\right) \rightarrow F_{\iota} \rightarrow \mathrm{D}_{n}^{t}(A) \stackrel{\iota}{\rightarrow} i_{n}^{t} A$ in $\mathcal{K}_{N}(R)$. Then

$$
\begin{gathered}
\operatorname{Hom}_{\mathcal{K}_{N}(R)}\left(C_{\iota}, X\right) \cong \operatorname{Hom}_{\mathcal{K}_{N}(R)}\left(C_{\iota}, X_{\leqslant n+N-1}\right)=0, \\
\operatorname{Hom}_{\mathcal{K}_{N}(R)}\left(F_{\iota}, X\right) \cong \operatorname{Hom}_{\mathcal{K}_{N}(R)}\left(F_{\iota}, X_{\leqslant n}\right)=0
\end{gathered}
$$


since $C_{\iota}, F_{\iota}$ are exact and $X_{\leqslant n+N-1}, X_{\leqslant n}$ are bounded above. Therefore,

$$
\operatorname{Hom}_{\mathcal{K}_{N}(R)}\left(i_{n}^{t} A, X\right) \cong \operatorname{Hom}_{\mathcal{K}_{N}(R)}\left(\mathrm{D}_{n}^{t}(A), X\right) .
$$

Now assume that $R$ is left noetherian and $A$ is finitely generated. Then $A$ is a compact object of $R$-Mod. By [6, p.688], we have $\operatorname{Hom}_{\mathcal{C}_{N}(R)}\left(\mathrm{D}_{n}^{t}(A), Y\right) \cong \operatorname{Hom}_{R}\left(A, \mathrm{Z}_{n}^{t}(Y)\right)$ for any $N$-complex $Y$. It follows that $\mathrm{D}_{n}^{t}(A)$ is a compact object of $\mathcal{K}_{N}(R)$ for all $n$ and $t$, and hence

$$
\begin{aligned}
\operatorname{Hom}_{\mathcal{K}_{N}(\mathscr{I})}\left(i_{n}^{t} A, \coprod_{\lambda \in \Lambda} X_{\lambda}\right) & \cong \operatorname{Hom}_{\mathcal{K}_{N}(R)}\left(\mathrm{D}_{n}^{t}(A), \coprod_{\lambda \in \Lambda} X_{\lambda}\right) \\
& \cong \coprod_{\lambda \in \Lambda} \operatorname{Hom}_{\mathcal{K}_{N}(R)}\left(\mathrm{D}_{n}^{t}(A), X_{\lambda}\right) \\
& \cong \coprod_{\lambda \in \Lambda} \operatorname{Hom}_{\mathcal{K}_{N}(\mathscr{I})}\left(i_{n}^{t} A, X_{\lambda}\right)
\end{aligned}
$$

for a family $\left\{X_{\lambda}\right\}_{\lambda \in \Lambda}$ in $\mathcal{K}_{N}(\mathscr{I})$. Therefore, $i_{n}^{t} A$ is a compact object of $\mathcal{K}_{N}(\mathscr{I})$ for all $n$ and $t$.

Theorem 4.8. Let $R$ be left noetherian. Then the category $\mathcal{K}_{N}(\mathscr{I})$ is compactly generated.

Proof. Each $N$-complex $i_{n}^{t} A$ is a compact object of $\mathcal{K}_{N}(\mathscr{I})$ by Lemma 4.7. It remains to show that $\mathscr{G}=\left\{i_{n}^{t} A \mid A \in R\right.$-Mod is finitely generated $\}$ is a set of generators of $\mathcal{K}_{N}(\mathscr{I})$. We claim that for any nonzero object $X$ of $\mathcal{K}_{N}(\mathscr{I}), \operatorname{Hom}_{\mathcal{K}_{N}(R)}(G, X) \neq 0$ for some $G \in \mathscr{G}$.

Suppose first that $\mathrm{H}_{n}^{t}(X) \neq 0$ for some $n$ and $t$. Choose a finitely generated left $R$-module $A$ and a nonzero homomorphism $A \rightarrow \mathrm{Z}_{n}^{t}(X)$. We obtain a nonzero chain map $\mathrm{D}_{n}^{t}(A) \rightarrow X$ that induces a nonzero element in $\operatorname{Hom}_{\mathcal{K}_{N}(R)}\left(\mathrm{D}_{n}^{t}(A), X\right)$. Suppose next that $\mathrm{H}_{n}^{t}(X)=0$ for all $n$ and $t$. We can choose $n$ and $t$ such that $\mathrm{Z}_{n}^{t}(X)$ is noninjective. Consider the exact sequence $0 \rightarrow \Omega X \rightarrow F_{i d_{X}} \rightarrow X \rightarrow 0$. Since $X$ is exact, $0 \rightarrow \mathrm{Z}_{n}^{t}(\Omega X) \rightarrow \mathrm{Z}_{n}^{t}\left(F_{i d_{X}}\right) \rightarrow \mathrm{Z}_{n}^{t}(X) \rightarrow 0$ is exact. Note that $F_{i d_{X}} \cong \prod_{n \in \mathbb{Z}} \mathrm{D}_{n}^{N}\left(X_{n}\right)$ by [16, Remark 2.15], so $\mathrm{Z}_{n}^{t}\left(F_{i d_{X}}\right)$ is injective. But $\mathrm{Z}_{n}^{t}(X)$ is non-injective, and it follows that $\mathrm{Z}_{n}^{t}(\Omega X)$ is non-injective. Using Baer's criterion, there is a finitely generated left $R$-module $A$ such that $\operatorname{Ext}_{R}^{1}\left(A, \mathrm{Z}_{n}^{t}(\Omega X)\right) \neq 0$. By Corollary 2.4, we have a commutative diagram:

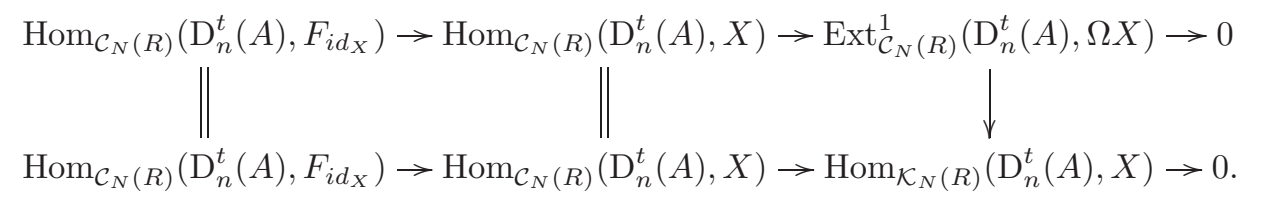

Also, $\operatorname{Ext}_{\mathcal{C}_{N}(R)}^{1}\left(\mathrm{D}_{n}^{t}(A), \Omega X\right) \cong \operatorname{Ext}_{R}^{1}\left(A, \mathrm{Z}_{n}^{t}(\Omega X)\right)$ by the proof of [6, Theorem 4.5]. Thus $\operatorname{Hom}_{\mathcal{K}_{N}(R)}\left(\mathrm{D}_{n}^{t}(A), X\right) \cong \operatorname{Ext}_{R}^{1}\left(A, \mathrm{Z}_{n}^{t}(\Omega X)\right) \neq 0$. This shows our claim. This implies that the category $\mathcal{K}_{N}(\mathscr{I})$ is compactly generated. 


\section{The homotopy category $\mathcal{K}_{N}(\mathscr{P})$}

In this section, $\mathscr{A}$ is the category of left $R$-modules and $\mathscr{P}$ the class of projectives. We denote the homotopy category of $N$-complexes in $\mathscr{P}$ by $\mathcal{K}_{N}(\mathscr{P})$. We show that the category $\mathcal{K}_{N}(\mathscr{P})$ is compactly generated when $R$ is a right coherent ring for which every flat left $R$-module has finite projective dimension.

Let $X$ be an $N$-complex. We define $X^{*}$ to be the $N$-complex

$$
\cdots \rightarrow \operatorname{Hom}_{R}\left(X_{n-1}, R\right) \rightarrow \operatorname{Hom}_{R}\left(X_{n}, R\right) \rightarrow \operatorname{Hom}_{R}\left(X_{n+1}, R\right) \rightarrow \cdots
$$

Construction 5.1. Let $R$ be right coherent and $M$ a finitely presented left $R$ module. By the proof of Proposition 3.4(1), we can choose a quasi-isomorphism $P \stackrel{\pi}{\rightarrow}\left(\mathrm{D}_{n}^{t}(M)\right)^{*}$ with $P$ bounded below and each $P_{l}$ finitely generated projective. There is also a canonical homomorphism $\mathrm{D}_{n}^{t}(M) \stackrel{\delta}{\rightarrow}\left(\mathrm{D}_{n}^{t}(M)\right)^{* *}$. Consider

$$
\mathrm{D}_{n}^{t}(M) \stackrel{\delta}{\longrightarrow}\left(\mathrm{D}_{n}^{t}(M)\right)^{* *} \stackrel{\pi^{*}}{\longrightarrow} P^{*} .
$$

If we consider $P^{*}$ and $P$ to be objects of $\mathcal{K}_{N}(\mathscr{P})$ and $\mathcal{K}_{N}(\mathscr{P}$ op $)$, then $P^{*}$ depends functorially on $P$, and $P$ depends functorially on $\left(\mathrm{D}_{n}^{t}(M)\right)^{*}$. But $\left(\mathrm{D}_{n}^{t}(M)\right)^{*}$ depends functorially on $\mathrm{D}_{n}^{t}(M)$, and so, altogether, $P^{*}$ depends functorially on $\mathrm{D}_{n}^{t}(M)$.

There exists a commutative diagram

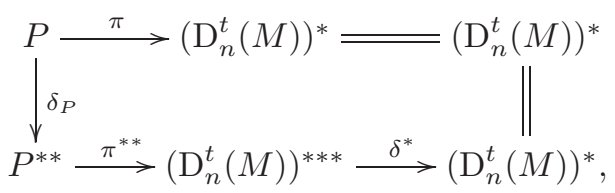

where $\delta_{P}$ is the canonical chain map. Since $P$ consists of finitely generated projective modules, $\delta_{P}$ is an isomorphism. Also, $\pi$ is a quasi-isomorphism, so the diagram shows that the composition $\delta^{*} \pi^{* *}$ is a quasi-isomorphism. That is,

$$
\mathrm{H}_{k}^{t^{\prime}}\left(\operatorname{Hom}_{R}\left(P^{*}, R\right)\right) \cong \mathrm{H}_{k}^{t^{\prime}}\left(\operatorname{Hom}_{R}\left(\mathrm{D}_{n}^{t}(M), R\right)\right), \forall k, t^{\prime} .
$$

But $M$ and each $P_{l}^{*}$ are finitely presented, and it follows that

$$
\mathrm{H}_{k}^{t^{\prime}}\left(\operatorname{Hom}_{R}\left(P^{*}, Q\right)\right) \cong \mathrm{H}_{k}^{t^{\prime}}\left(\operatorname{Hom}_{R}\left(\mathrm{D}_{n}^{t}(M), Q\right)\right), \forall k, t^{\prime}
$$

for any projective left $R$-module $Q$. Consider the left and right triangles in $\mathcal{K}_{N}(R)$ :

$$
\Omega P^{*} \rightarrow F \rightarrow \mathrm{D}_{n}^{t}(M) \stackrel{\pi^{*} \delta}{\rightarrow} P^{*}, \mathrm{D}_{n}^{t}(M) \stackrel{\pi^{*} \delta}{\rightarrow} P^{*} \rightarrow C \rightarrow \Sigma\left(\mathrm{D}_{n}^{t}(M)\right) .
$$

By the preceding proof, $\operatorname{Hom}_{\mathcal{K}_{N}(R)}\left(F, \mathrm{D}_{n}^{1}(Q)\right)=\operatorname{Hom}_{\mathcal{K}_{N}(R)}\left(C, \mathrm{D}_{n}^{1}(Q)\right)=0$ for any projective left $R$-module $Q$ and all $n$.

Lemma 5.2. Assume that $X, Y \in \mathcal{C}_{N}(R)$ with either $X$ bounded below or $Y$ bounded below. If $\operatorname{Hom}_{\mathcal{K}_{N}(R)}\left(\mathrm{D}_{k}^{1}\left(X_{k}\right), Y\right)=0$ for all $k$, then $\operatorname{Hom}_{\mathcal{K}_{N}(R)}(X, Y)=0$.

Proof. We need to show that every chain map $\alpha: X \rightarrow Y$ is null-homotopic. Thus for a given $\alpha$ we must construct a collection of morphisms $s_{m}: X_{m} \rightarrow Y_{m+N-1}$, such that

$$
\alpha_{m}=d^{N-1} s_{m}+d^{N-2} s_{m-1} d+\cdots+s_{m-N+1} d^{N-1} \quad\left(*_{m}\right)
$$

for all $m$. We do so by induction on $m$. Since $X$ or $Y$ is bounded below, we must have $s_{m}=0$ for $m \ll 0$. For the inductive step, assume that $s_{m}$ has been constructed for 
all $m<\tilde{m}$. Using that $\alpha$ is a chain map and that $\left(*_{\tilde{m}-1}\right)$ holds, we see that

$$
\begin{aligned}
& d_{\tilde{m}}^{Y}\left(\alpha_{\tilde{m}}-\left(d^{N-2} s_{\tilde{m}-1} d+\cdots+d s_{\tilde{m}-N+2} d^{N-2}+s_{\tilde{m}-N+1} d^{N-1}\right)\right) \\
& =\left(\alpha_{\tilde{m}-1}-d_{\tilde{m}}^{Y}\left(d^{N-2} s_{\tilde{m}-1}+\cdots+d s_{\tilde{m}-N+2} d^{N-3}+s_{\tilde{m}-N+1} d^{N-2}\right)\right) d_{\tilde{m}}^{X} \\
& =\left(s_{\tilde{m}-N} d^{N-1}\right) d_{\tilde{m}}^{X}=0 .
\end{aligned}
$$

But $\operatorname{Hom}_{\mathcal{K}_{N}(R)}\left(\mathrm{D}_{\tilde{m}}^{1}\left(X_{\tilde{m}}\right), Y\right)=0$, and it follows that there exists $s_{\tilde{m}}: X_{\tilde{m}} \rightarrow Y_{\tilde{m}+N-1}$ such that $d^{N-1} s_{\tilde{m}}=\alpha_{\tilde{m}}-\left(d^{N-2} s_{\tilde{m}-1} d+\cdots+s_{\tilde{m}-N+1} d^{N-1}\right)$, as claimed.

Similarly, one establishes the next lemma.

Lemma 5.3. Assume that $X, Y \in \mathcal{C}_{N}(R)$ with either $X$ bounded above or $Y$ bounded above. If $\operatorname{Hom}_{\mathcal{K}_{N}(R)}\left(X, \mathrm{D}_{k}^{1}\left(Y_{k}\right)\right)=0$ for all $k$, then $\operatorname{Hom}_{\mathcal{K}_{N}(R)}(X, Y)=0$.

Remark 5.4. Let $R$ be right coherent and $A$ a finitely presented left $R$-module. Then Construction 5.1 provided a quasi-isomorphism $\mathrm{D}_{n}^{t}(A) \stackrel{\widetilde{\sim}}{\rightarrow} P$ with $P$ a bounded-above $N$-complex of finitely generated projective left $R$-modules for any $n$ and $t$. We denote the $N$-complex $P$ by $p_{n}^{t} A$.

Lemma 5.5. Let $R$ be right coherent and $A$ a finitely presented left $R$-module. Then for any $X \in \mathcal{K}_{N}(\mathscr{P})$ we have an isomorphism

$$
\operatorname{Hom}_{\mathcal{K}_{N}(R)}\left(p_{n}^{t} A, X\right) \cong \mathrm{H}_{n}^{t}\left(\operatorname{Hom}_{R}(A, X)\right), \forall n, t .
$$

In particular, $p_{n}^{t} A$ is a compact object in $\mathcal{K}_{N}(\mathscr{P})$.

Proof. By Lemma 5.3, $\operatorname{Hom}_{\mathcal{K}_{N}(R)}(F, X)=\operatorname{Hom}_{\mathcal{K}_{N}(R)}(C, X)=0$, where $F, C$ are as in Construction 5.1. Hence $\operatorname{Hom}_{\mathcal{K}_{N}(\mathscr{P})}\left(p_{n}^{t} A, X\right) \cong \operatorname{Hom}_{\mathcal{K}_{N}(R)}\left(\mathrm{D}_{n}^{t}(A), X\right)$ for any $n$ and $t$. Note that $\operatorname{Hom}_{\mathcal{K}_{N}(R)}\left(\mathrm{D}_{n}^{t}(A), X\right) \cong \mathrm{H}_{n}^{t}\left(\operatorname{Hom}_{R}(A, X)\right)$. This shows the desired isomorphism. For the last claim, we use that $\mathrm{H}_{n}^{t}\left(\operatorname{Hom}_{R}(A,-)\right)$ commutes with setindexed coproducts.

The following result was proved by Jøgensen when $N=2$ (see [10, Theorem 2.4]).

Theorem 5.6. Let $R$ be a right coherent ring for which every flat left $R$-module has finite projective dimension. Then the category $\mathcal{K}_{N}(\mathscr{P})$ is compactly generated.

Proof. Each $N$-complex $p_{n}^{t} A$ is a compact object in $\mathcal{K}_{N}(\mathscr{P})$ by Lemma 5.5. It remains to show that $\mathscr{G}=\left\{p_{n}^{t} A \mid A \in R\right.$-Mod is finitely presented $\}$ is a set of generators of $\mathcal{K}_{N}(\mathscr{P})$. So suppose that $X$ in $\mathcal{K}_{N}(\mathscr{P})$ has $\operatorname{Hom}_{\mathcal{K}_{N}(\mathscr{P})}(G, X)=0$ for every $G \in \mathscr{G}$. We show that $X \cong 0$ in $\mathcal{K}_{N}(\mathscr{P})$.

First $0=\operatorname{Hom}_{\mathcal{K}_{N}(\mathscr{P})}\left(p_{n}^{t} R, X\right) \cong \mathrm{H}_{n}^{t}\left(\operatorname{Hom}_{R}(R, X)\right) \cong \mathrm{H}_{n}^{t}(X)$ for all $n$ and $t$. So $X$ is exact. Again by Lemma 5.5, for any finitely presented left $R$-module $A$,

$$
0=\operatorname{Hom}_{\mathcal{K}_{N}(\mathscr{P})}\left(p_{n}^{t} A, X\right) \cong \mathrm{H}_{n}^{t}\left(\operatorname{Hom}_{R}(A, X)\right), \forall n, t .
$$

Hence [8, Theorem 6.4] implies that $0 \rightarrow \mathrm{Z}_{n}^{t}(X) \rightarrow X_{n} \rightarrow \mathrm{Z}_{n-t}^{N-t}(X) \rightarrow 0$ is pure exact and $\mathrm{Z}_{n}^{t}(X)$ is flat for all $n$ and $t$. Finally, note that $\operatorname{pd}_{R} \mathrm{Z}_{n-s}^{N-1}(X) \leqslant s$ for some $s$ by assumption and the sequence $0 \rightarrow \mathrm{C}_{n}^{N-1}(X) \rightarrow X_{n-1} \rightarrow \cdots \rightarrow X_{n-s+1} \rightarrow \mathrm{Z}_{n-s}^{N-1}(X) \rightarrow$ 0 is exact; it follows that $\mathrm{C}_{n}^{N-1}(X)$ is projective, and so $\mathrm{Z}_{n}^{1}(X)$ is projective for each 
$n$. Note that $0 \rightarrow \mathrm{Z}_{n+N-t}^{N-t}(X) \rightarrow \mathrm{Z}_{n+N-t}^{N-t+1}(X) \stackrel{d^{N-t}}{\rightarrow} \mathrm{Z}_{n}^{1}(X) \rightarrow 0$ is exact, so $\mathrm{Z}_{n}^{t}(X)$ is projective for all $n$ and $t$ by induction. Thus $X$ is a projective $N$-complex by the dual of $\left[\mathbf{4}\right.$, Theorem 4.5], and hence $X \cong 0$ in $\mathcal{K}_{N}(\mathscr{P})$, as desired.

\section{Recollements}

Let $\mathscr{L}$ be a class of left $R$-modules. We denote the full subcategory formed by all exact $N$-complexes in $\mathcal{K}_{N}(\mathscr{L})$ by $\mathcal{K}_{N}^{e x}(\mathscr{L})$. In this section, We establish a recollement of the pretriangulated category $\mathcal{K}_{N}(R)$ relative to $\mathcal{K}_{N}^{e x}(R)$ and $\mathcal{D}_{N}(R)$ and an equivalence $\mathcal{K}_{N}^{c}(\mathscr{I}) \rightarrow \mathcal{D}_{N}^{b}(R \bmod )$.

If $\left(\mathscr{T}_{1}, \Omega_{1}, \Delta_{1}\right)$ and $\left(\mathscr{T}_{2}, \Omega_{2}, \Delta_{2}\right)$ are left triangulated categories, then a functor $F: \mathscr{T}_{1} \rightarrow \mathscr{T}_{2}$ is called left exact if there is a natural isomorphism $\xi: \Omega_{2} F \stackrel{\cong}{\Rightarrow} F \Omega_{1}$ such that for any left triangle $\Omega_{1} Z \stackrel{\mu}{\rightarrow} X \stackrel{\nu}{\rightarrow} Y \stackrel{\omega}{\rightarrow} Z$ in $\mathscr{T}_{1}$, the diagram $\Omega_{2}(F(Z)) \stackrel{F(\mu) \xi_{Z}}{\rightarrow}$ $F(X) \stackrel{F(\nu)}{\rightarrow} F(Y) \stackrel{F(\omega)}{\rightarrow} F(Z)$ is a left triangle in $\mathscr{T}_{2}$. Similarly one defines right exact functors between right triangulated categories. Let $H: \mathscr{T}_{1} \rightarrow \mathscr{T}_{2}$ be a functor between pretriangulated categories. Then $H$ is called exact if $H$ is left and right exact.

Let $\mathscr{T}^{\prime}, \mathscr{T}$ and $\mathscr{T}^{\prime \prime}$ be pretriangulated categories. The diagram of exact functors

$$
\mathscr{T}^{\prime} \stackrel{i_{*}}{\longrightarrow} \mathscr{T} \stackrel{j^{*}}{\longrightarrow} \mathscr{T}^{\prime \prime}
$$

is a recollement of $\mathscr{T}$ relative to $\mathscr{T}^{\prime}$ and $\mathscr{T}^{\prime \prime}$, if the following conditions are satisfied:

(R1) The functor $i_{*}$ is fully faithful and has a left adjoint $i^{*}$ and a right adjoint $i^{\prime}$;

(R2) The functor $j^{*}$ has a fully faithful left adjoint $j$ ! and a fully faithful right adjoint $j_{*}$;

(R3) $\operatorname{Im} i_{*}=\operatorname{Ker} j^{*}$.

Theorem 6.1. Let $R$ be a ring. We have the following recollement:

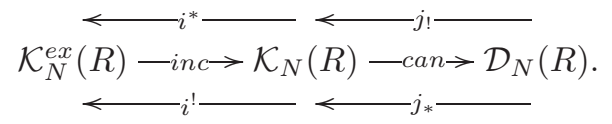

Moreover, the composition $d g \widetilde{\mathscr{P}}_{N} \stackrel{j}{\rightarrow} \mathcal{K}_{N}(R) \stackrel{Q}{\rightarrow} \mathcal{D}_{N}(R)$ is an equivalence of right triangulated categories, the composition $d g \widetilde{\mathscr{I}}_{N} \stackrel{j^{\prime}}{\rightarrow} \mathcal{K}_{N}(R) \stackrel{Q}{\rightarrow} \mathcal{D}_{N}(R)$ is an equivalence of left triangulated categories.

Proof. We first construct the functors involved. Let $X$ be an $N$-complex. Then Theorem 3.8 yields a quasi-isomorphism $s: P \stackrel{\widetilde{\rightrightarrows}}{\rightarrow} X$ with $P \in d g \widetilde{\mathscr{P}}_{N}$, and we have a right triangle $P \stackrel{s}{\rightarrow} X \rightarrow C_{s} \rightarrow \Sigma P$ with $C_{s} \in \mathcal{K}_{N}^{e x}(R)$. Suppose that $t: P^{\prime} \stackrel{\widetilde{\rightrightarrows}}{\rightarrow} X$ with $P^{\prime} \in d g \widetilde{P}_{N}$. Then we have a commutative diagram:

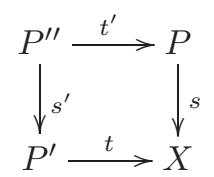

such that $P^{\prime \prime} \in d g \widetilde{P}_{N}$ and $s^{\prime}, t^{\prime}$ are quasi-isomorphisms. Thus we have the following 
morphism of right triangles in $\mathcal{K}_{N}(R)$ :

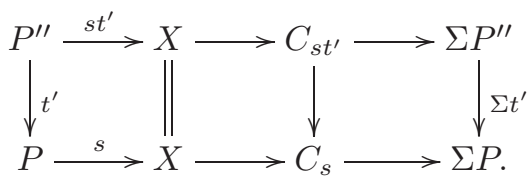

Note that $t^{\prime}$ is a homotopy equivalence; it follows that $C_{s} \cong C_{s t^{\prime}}$ in $\mathcal{K}_{N}^{e x}(R)$. Similarly, we have $C_{t} \cong C_{t s^{\prime}}=C_{s t^{\prime}}$ in $\mathcal{K}_{N}^{e x}(R)$. Hence $C_{t} \cong C_{s}$ in $\mathcal{K}_{N}^{e x}(R)$. On the other hand, let $0 \sim f \in \operatorname{Hom}_{\mathcal{K}_{N}(R)}(X, Y)$. Then we have a morphism of right triangles in $\mathcal{K}_{N}(R)$ :

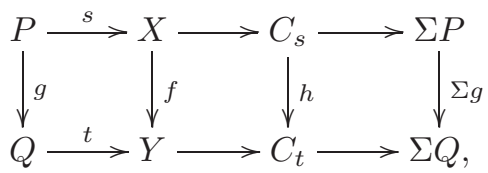

where $P, Q \in d g \widetilde{P}_{N}$ and $s, t$ are quasi-isomorphisms. We obtain $g \sim 0$, and hence $h \sim 0$. This implies that $i^{*}: \mathcal{K}_{N}(R) \rightarrow \mathcal{K}_{N}^{e x}(R)$ given by $i^{*}(X)=C_{s}$ is a functor. Let $E$ be an exact $N$-complex. Then we have an exact sequence

$$
\operatorname{Hom}_{\mathcal{K}_{N}(R)}(\Sigma P, E) \rightarrow \operatorname{Hom}_{\mathcal{K}_{N}(R)}\left(C_{s}, E\right) \rightarrow \operatorname{Hom}_{\mathcal{K}_{N}(R)}(X, E) \rightarrow \operatorname{Hom}_{\mathcal{K}_{N}(R)}(P, E)=
$$

But $\operatorname{Hom}_{\mathcal{K}_{N}(R)}(\Sigma P, E) \cong \operatorname{Hom}_{\mathcal{K}_{N}(R)}(P, \Omega E)=0$; it follows that

$$
\operatorname{Hom}_{\mathcal{K}_{N}^{e x}(R)}\left(i^{*}(X), E\right)=\operatorname{Hom}_{\mathcal{K}_{N}(R)}\left(C_{s}, E\right) \cong \operatorname{Hom}_{\mathcal{K}_{N}(R)}(X, E) .
$$

This shows that $\left(i^{*}, i n c\right)$ is an adjoint pair.

Let $X$ be an $N$-complex. Then Theorem 3.11 yields a quasi-isomorphism $s: X \stackrel{\simeq}{\rightarrow} I$ with $I \in d g \widetilde{\mathscr{I}}_{N}$, and we have a left triangle $\Omega I \rightarrow F_{s} \rightarrow X \stackrel{s}{\rightarrow} I$ with $F_{s} \in \mathcal{K}_{N}^{e x}(R)$. By analogy with the preceding proof, we see that $i^{!}: \mathcal{K}_{N}(R) \rightarrow \mathcal{K}_{N}^{e x}(R)$ given by $i^{!}(X)=F_{s}$ is a functor. Let $E \in \mathscr{E}_{N}$. Then we have an exact sequence

$$
\operatorname{Hom}_{\mathcal{K}_{N}(R)}(E, \Omega I) \rightarrow \operatorname{Hom}_{\mathcal{K}_{N}(R)}\left(E, F_{s}\right) \rightarrow \operatorname{Hom}_{\mathcal{K}_{N}(R)}(E, X) \rightarrow \operatorname{Hom}_{\mathcal{K}_{N}(R)}(E, I) .
$$

Therefore, $\operatorname{Hom}_{\mathcal{K}_{N}(R)}(E, X) \cong \operatorname{Hom}_{\mathcal{K}_{N}^{e x}(R)}\left(E, i^{!}(X)\right)$ and $\left(\right.$ inc,$\left.i^{!}\right)$is an adjoint pair.

Let $X$ be an $N$-complex. Then Theorem 3.8 yields a quasi-isomorphism $s: P \stackrel{\simeq}{\rightrightarrows} X$ with $P \in d g \widetilde{\mathscr{P}}_{N}$. On the other hand, let $0 \simeq f \in \operatorname{Hom}_{\mathcal{D}_{N}(R)}(X, Y)$. Then we have a commutative diagram in $\mathcal{K}_{N}(R)$ :

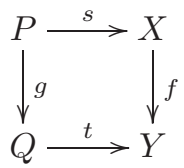

where $P, Q \in d g \widetilde{P}_{N}$ and $s, t$ are quasi-isomorphisms. We obtain $g \simeq 0$. This implies that $j_{!}: \mathcal{D}_{N}(R) \rightarrow \mathcal{K}_{N}(R)$ given by $j_{!}(X)=P$ is a functor. Let $X, Y$ be $N$-complexes and $P \stackrel{\simeq}{\rightrightarrows} X, Q \stackrel{\simeq}{\rightrightarrows} Y$ with $P, Q \in d g \widetilde{\mathscr{P}}_{N}$. Then Lemma 3.3 implies that

$$
\operatorname{Hom}_{\mathcal{D}_{N}(R)}(X, Y) \cong \operatorname{Hom}_{\mathcal{D}_{N}(R)}(P, Q) \cong \operatorname{Hom}_{\mathcal{K}_{N}(R)}\left(j_{!}(X), j_{!}(Y)\right)
$$


which shows that $j_{\text {! }}$ is fully faithful. Also, we have the isomorphisms

$$
\operatorname{Hom}_{\mathcal{K}_{N}(R)}(j !(X), Y)=\operatorname{Hom}_{\mathcal{K}_{N}(R)}(P, Y) \cong \operatorname{Hom}_{\mathcal{D}_{N}(R)}(P, Y) \cong \operatorname{Hom}_{\mathcal{D}_{N}(R)}(X, Y) .
$$

This implies that $(j !, c a n)$ is an adjoint pair.

Let $X$ be an $N$-complex. Then Theorem 3.11 yields a quasi-isomorphism $X \stackrel{\simeq}{\rightarrow} I$ with $I \in d g \widetilde{\mathscr{I}_{N}}$. By analogy with the preceding proof, we see that $j_{*}: \mathcal{D}_{N}(R) \rightarrow$ $\mathcal{K}_{N}(R)$ given by $j_{*}(X)=I$ is a fully faithful functor and $\left(\right.$ can,$\left.j_{*}\right)$ is an adjoint pair. We have established the desired recollement.

By [3, Lemma 2.1], $d g \widetilde{\mathscr{P}}_{N}$ is a right triangulated subcategory of $\mathcal{K}_{N}(R)$. So the inclusion $j: d g \widetilde{\mathscr{P}}_{N} \rightarrow \mathcal{K}_{N}(R)$ is a right exact functor. This yields a functor $Q j$ : $d g \widetilde{P}_{N} \rightarrow \mathcal{D}_{N}(R)$ that is distinct on objects, and this functor is fully faithful by Lemma 3.3. Let $X, Y$ be objects of $\mathcal{K}_{N}(R)$. Note that $j$ ! sends every object of $\mathcal{D}_{N}(R)$ into an object of $d g \widetilde{P}_{N} \subseteq \mathcal{K}_{N}(R)$; it follows from Lemma 3.3 that

$$
\operatorname{Hom}_{\mathcal{D}_{N}(R)}(X, Q(Y)) \cong \operatorname{Hom}_{\mathcal{K}_{N}(R)}(j !(X), Y) \cong \operatorname{Hom}_{\mathcal{D}_{N}(R)}(Q j !(X), Q(Y)) .
$$

One checks that this map is just composition with the counit $\varepsilon_{X}: Q j_{!}(X) \rightarrow X$, and by a standard argument this implies that $\varepsilon_{X}$ is an isomorphism. Therefore, $Q j$ is an equivalence. The argument for the second equivalence is identical.

Proposition 6.2. Let $R$ be a left noetherian ring, and let $\mathcal{D}_{N}^{b}(\bmod )$ denote the full subcategory of objects $X$ in $\mathcal{D}_{N}(R)$ such that $H_{n}^{t}(X)$ is finitely generated for all $n, t$ and $H_{n}^{t}(X)=0$ for almost all $n, t$. Then the canonical functor $Q: \mathcal{K}_{N}(R) \rightarrow \mathcal{D}_{N}(R)$ induces an equivalence

$$
\mathcal{K}_{N}^{c}(\mathscr{I}) \longrightarrow \mathcal{D}_{N}^{b}(\bmod ) .
$$

Proof. It follows from Theorem 4.8 that $\mathcal{K}_{N}(\mathscr{I})$ is compactly generated by the set $\mathscr{G}=\left\{i_{n}^{t} A \mid A \in R\right.$-Mod is finitely generated $\}$. Let $A \in R$-Mod be finitely generated. Consider the split exact sequence

$$
0 \longrightarrow \mathrm{D}_{n-t}^{N-t}(A) \longrightarrow \mathrm{D}_{n}^{N}(A) \longrightarrow \mathrm{D}_{n}^{t}(A) \longrightarrow 0 .
$$

Then we have a left triangle $\Omega \mathrm{D}_{n}^{t}(A) \rightarrow \mathrm{D}_{n-t}^{N-t}(A) \rightarrow \mathrm{D}_{n}^{N}(A) \rightarrow \mathrm{D}_{n}^{t}(A)$ in $\mathcal{K}_{N}(R)$ by [16, Theorem 2.22]. Also, Proposition 3.5 yields a commutative diagram in $\mathcal{K}_{N}(R)$

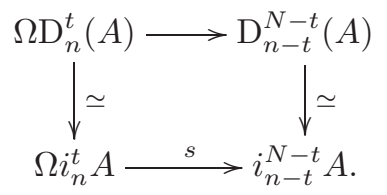

Since $\mathrm{D}_{n}^{N}(A)$ is exact, $\Omega \mathrm{D}_{n}^{t} A \rightarrow \mathrm{D}_{n-t}^{N-t} A$ is a quasi-isomorphism, and hence $s$ is so. Let $X$ be an object in $\mathcal{K}_{N}(\mathscr{I})$. Then $\operatorname{Hom}_{\mathcal{K}_{N}(R)}\left(F_{s}, \mathrm{D}_{k}^{1}\left(X_{k}\right)\right)=0=\operatorname{Hom}_{\mathcal{K}_{N}(R)}\left(C_{s}, \mathrm{D}_{k}^{1}\right.$ $\left(X_{k}\right)$ ) for all $k$. But $F_{s}$ and $C_{s}$ are bounded above, so $\operatorname{Hom}_{\mathcal{K}_{N}(R)}\left(F_{s}, X\right)=0=$ $\operatorname{Hom}_{\mathcal{K}_{N}(R)}\left(C_{s}, X\right)$ by Lemma 5.3. Thus

$$
\operatorname{Hom}_{\mathcal{K}_{N}(\mathscr{I})}\left(\Omega i_{n}^{t}(A), X\right) \cong \operatorname{Hom}_{\mathcal{K}_{N}(\mathscr{I})}\left(i_{n-t}^{N-t} A, X\right) .
$$


Now let $\left\{X_{\lambda}\right\}_{\lambda \in \Lambda}$ be a family of objects in $\mathcal{K}_{N}(\mathscr{I})$. Then

$$
\begin{aligned}
\operatorname{Hom}_{\mathcal{K}_{N}(\mathscr{I})}\left(\Omega i_{n}^{t}(A), \coprod_{\lambda \in \Lambda} X_{\lambda}\right) & \cong \operatorname{Hom}_{\mathcal{K}_{N}(\mathscr{I})}\left(i_{n-t}^{N-t} A, \coprod_{\lambda \in \Lambda} X_{\lambda}\right) \\
& \cong \coprod_{\lambda \in \Lambda} \operatorname{Hom}_{\mathcal{K}_{N}(\mathscr{I})}\left(i_{n-t}^{N-t} A, X_{\lambda}\right) \\
& \cong \coprod_{\lambda \in \Lambda} \operatorname{Hom}_{\mathcal{K}_{N}(\mathscr{I})}\left(\Omega i_{n}^{t}(A), X_{\lambda}\right) .
\end{aligned}
$$

This implies that $\Omega i_{n}^{t}(A) \in \mathcal{K}_{N}^{c}(\mathscr{I})$ for all $n$ and $t$, and $\mathcal{K}_{N}^{c}(\mathscr{I})$ is a thick subcategory of $\mathcal{K}_{N}(\mathscr{I})$ that is generated by the injective resolutions of the finitely generated left $R$-modules. Also, Lemmas 3.2 and 3.3 and Proposition 3.4 imply that the canonical functor $\mathcal{K}_{N}^{-}(\mathscr{I}) \rightarrow \mathcal{D}_{N}^{-}(R)$ is an equivalence, which restricts to an equivalence $\mathcal{K}_{N}^{-, b}(\mathscr{I}) \rightarrow \mathcal{D}_{N}^{b}(R)$ and identifies $\mathcal{K}_{N}^{c}(\mathscr{I})$ with $\mathcal{D}_{N}^{b}(\bmod )$.

\section{Acknowledgments}

This research was partially supported by National Natural Science Foundation of China $(11261050,11361051,11361052)$ and Program for New Century Excellent Talents in University (NCET-13-0957). We wish to thank the referee for the very helpful suggestions that have been incorporated herein.

\section{References}

[1] I. Assem, A. Beligiannis, and N. Marmaridis, Right triangulated categories with right semiequivalence, Algebras and Modules II (Geiranger, 1996), CMS Conf. Proc. 24 Amer. Math. Soc. Providence, RI (1998), 17-37.

[2] A. Beligiannis and N. Marmaridis, Left triangulated categories arising from contravariantly finite subcategories, Comm. Algebra 22 (1994), 5021-5036.

[3] A. Beligiannis and I. Reiten, Homological and homotopical aspects of torsion theories, Mem. Amer. Math. Soc, 2007.

[4] S. Estrada, Monomial algebras over infinite quivers. Applications to $N$-complexes of modules, Comm. Algebra 35 (2007), 3214-3225.

[5] J. Gillespie, The homotopy category of $N$-complexes is a homotopy category, J. Homotopy Relat. Struct. (2013), 1-12.

[6] J. Gillespie and M. Hovey, Gorenstein model structures and generalized derived categories, Proc. Edinb. Math. Soc. 53 (2010), 675-696.

[7] M. Hoshino, Derived categories, http://www.u-gakugei.ac.jp/ miyachi/papers/DC1.pdf.

[8] C.U. Jensen and H. Lenzing, Model-theoretic algebra with particular emphasis on fields, rings, modules, Vol. 2, Algebra Logic Appl., Gordon and Breach Science Publishers, New York, 1989.

[9] S. Iyengar and H. Krause, Acyclicity versus total acyclicity for complexes over Noetherian rings, Documenta Math. 11 (2006), 207-240.

[10] P. Jøgensen, The homotopy category of complexes of projective modules, Adv. Math. 193 (2005), $223-232$.

[11] M.M. Kapranov, On the q-analog of homological algebra, Preprint, arXiv:q-alg/9611005 (1996).

[12] H. Krause, The stable derived category of a noetherian scheme, Compositio Math. 141 (2005), $1128-1162$.

[13] D. Murfet, Derived categories part I, http://therisingsea.org/notes/DerivedCategories.pdf, October 5, 2006

[14] _ Abelian categories, http://therisingsea.org/notes/AbelianCategories.pdf, October 5, 2006.

[15] A. Neeman, The homotopy category of flat modules and Grothendieck duality, Invent. Math. 174 (2008), 255-308. 
[16] X.Y. Yang and N.Q. Ding, The homotopy category and derived category of $N$-complexes, J. Algebra 426 (2015), 430-476.

Xiaoyan Yang yangxy@nwnu.edu.cn

Department of Mathematics, Northwest Normal University, Lanzhou 730070, China

Junpeng Wang wangjunpeng1218@163.com

Department of Mathematics, Northwest Normal University, Lanzhou 730070, China 